\title{
On interval predictor-corrector methods
}

\author{
Andrzej Marciniak ${ }^{1,2}$ • Malgorzata A. Jankowska ${ }^{3}$. \\ Tomasz Hoffmann 4
}

Received: 26 March 2016 / Accepted: 3 October 2016 / Published online: 13 October 2016 (C) The Author(s) 2016. This article is published with open access at Springerlink.com

\begin{abstract}
One can approximate numerically the solution of the initial value problem using single or multistep methods. Linear multistep methods are used very often, especially combinations of explicit and implicit methods. In floating-point arithmetic from an explicit method (a predictor), we can get the first approximation to the solution obtained from an implicit method (a corrector). We can do the same with interval multistep methods. Realizing such interval methods in floating-point interval arithmetic, we compute solutions in the form of intervals which contain all possible errors. In this paper, we propose interval predictor-corrector methods based on conventional Adams-Bashforth-Moulton and Nyström-Milne-Simpson methods. In numerical examples, these methods are compared with interval methods of RungeKutta type and methods based on high-order Taylor series. It appears that the presented methods yield comparable approximations to the solutions.
\end{abstract}

Malgorzata A. Jankowska

malgorzata.jankowska@put.poznan.pl

Andrzej Marciniak

andrzej.marciniak@put.poznan.pl

Tomasz Hoffmann

tomhof@man.poznan.pl

1 Institute of Computing Science, Poznan University of Technology, Piotrowo 2, 60-965

Poznan, Poland

2 Department of Computer Science, Higher Vocational State School in Kalisz, Poznanska 201-205, 62-800 Kalisz, Poland

3 Institute of Applied Mechanics, Poznan University of Technology, Jana Pawła II 24, 60-965

Poznan, Poland

4 Poznan Supercomputing and Networking Center, Jana Pawła II 10, 61-139 Poznan, Poland 
Keywords Initial value problem $\cdot$ Multistep methods $\cdot$ Interval predictor-corrector methods · Floating-point interval arithmetic

\section{Introduction}

Many scientific and engineering problems are described in the form of ordinary differential equations. If such equations cannot be solved analytically, we use computers and numerical methods to approximate them, usually performing all calculations in floating-point arithmetic. Sometimes, although in rare circumstances, this arithmetic is error-prone. Therefore, particular attention must be paid to the reliability of the computed results.

It is well-known that floating-point arithmetic is prone to two kinds of errors: representation errors and rounding errors. Applying numerical methods to approximate problems given by ordinary differential equations, we introduce third kind of errorsthe errors of methods, usually called truncation errors. To take into consideration these errors, we can use interval arithmetic (see, e.g., [1, 14, 33, 34, 41]) realized in floating-point interval arithmetic (see, e.g., [13]). Applying interval methods in such an arithmetic, we can obtain approximated solutions in the form of intervals which contain all possible numerical errors. One inconvenience of this arithmetic is the wrapping effect, but some efforts reduce its effects (see, e.g., [12, 23, 24, 37, 39, 42]).

There are a number of interval methods for approximating the initial value problem which consists in an ordinary differential equation (or equations) and an initial value (or values for a system of equations) of the function(s) that should be found. An interval method for ordinary differential equations using interval arithmetic was described first by R. E. Moore in 1965 [32, 33]. There are also interval methods based on explicit Runge-Kutta methods [21, 28, 41] and implicit ones [10, 11, 25, 28, 31]. In [41], Yu. I. Shokin proposed an explicit interval method of Adams-Bashforth type. Unfortunately, it can be shown that his formula fails in the simplest case, but it can be easily corrected $[19,28]$. Other explicit interval multistep methods have been considered in [26-28] and implicit ones in [18, 20, 26-28]. In recent years, many studies have been conducted on a variety of interval methods based on high-order Taylor series (see, e.g., [2-4, 7, 17, 22, 36, 38, 40]).

In this paper, we propose to join explicit interval methods of Adams-Bashforth type with implicit ones of Adams-Moulton type and explicit interval methods of Nyström type with implicit ones of Milne-Simpson type. In both cases, we obtain interval predictor-corrector methods, which have the same advantage as conventional predictor-corrector methods. In comparison when we use the solution obtained for the previous integration step as the first approximation in an implicit method, taking the solution from an explicit interval multistep method (a predictor) as such an approximation, we can significantly reduce the number of iterations needed in the implicit method (a corrector).

The paper is divided into seven sections. In Section 2, we recall the well-known conventional multistep methods of Adams-Bashforth, Adams-Moulton, Nyström, and Milne-Simpson. Interval versions of explicit multistep methods are presented in Section 3, and implicit interval multistep methods are described in Section 4. In these 
sections, we point to some important theorems proved in our previous papers. Propositions for combining explicit and implicit interval multistep methods that lead to interval predictor-corrector methods are described briefly in Section 5. In Section 6, we present a number of numerical examples, and in the last section, some conclusions are given.

\section{Conventional predictor-corrector methods}

The numerical methods for approximating the initial value problem

$$
y^{\prime}=f(t, y(t)), \quad y(0)=y_{0},
$$

where $t \in[0, a], y \in \mathbb{R}^{N}$, and $f:[0, a] \times \mathbb{R}^{N} \rightarrow \mathbb{R}^{N}$ are called multistep methods if the approximation of $y(t)$ at $t=t_{k}$ uses the values of the dependent variable (and its derivative) at more than one mesh point.

To construct the well-known Adams-Bashforth and Adams-Moulton multistep methods, we rewrite the problem (1) in the equivalent integral form

$$
y(t)=y\left(t_{k-1}\right)+\int_{t_{k-1}}^{t} f(\tau, y(\tau)) d \tau, \quad t>t_{k-1},
$$

from which we have

$$
y\left(t_{k}\right)=y\left(t_{k-1}\right)+\int_{t_{k-1}}^{t_{k}} f(\tau, y(\tau)) d \tau
$$

To obtain multistep methods, we approximate the function $f(\tau, y(\tau))$ by an adequate interpolation polynomial, and we integrate this polynomial. Taking the polynomial $P(\tau)$ of the degree $n-1$ such that

$$
P\left(t_{k-j}\right)=f\left(t_{k-j}, y\left(t_{k-j}\right)\right), \quad j=1,2, \ldots, n,
$$

and assuming that we have already obtained approximate values $y_{k-n}$, $y_{k-n+1}, \ldots, y_{k-1}$ of $y\left(t_{k-n}\right), y\left(t_{k-n+1}\right), \ldots, y\left(t_{k-1}\right)$, respectively, we can write the following approximate formula for $y\left(t_{k}\right)$ :

$$
y_{k}=y_{k-1}+h \sum_{j=0}^{n-1} \gamma_{j} \nabla^{j} f\left(t_{k-1}, y_{k-1}\right)
$$

where $h=t_{i}-t_{i-1}$ for each $i=k-n+1, k-n+2, \ldots, n$,

$$
\nabla^{j} f\left(t_{k-1}, y_{k-1}\right)=\sum_{m=0}^{j}(-1)^{m}\left(\begin{array}{c}
j \\
m
\end{array}\right) f\left(t_{k-1-m}, y_{k-1-m}\right),
$$


(the symbol $\nabla$ denotes the backward difference operator), and

$$
\gamma_{0}=1, \quad \gamma_{j}=\frac{1}{j !} \int_{0}^{1} s(s+1) \cdots(s+j-1) d s \text { for } j=1,2, \ldots, n .
$$

The formula (3) is known as the $n$-step explicit Adams-Bashforth method, for which the truncation error has form

$$
\begin{aligned}
h^{n+1} \gamma_{n} \psi(\eta, y(\eta)) & \equiv h^{n+1} \gamma_{n} f^{(n)}(\eta, y(\eta)) \\
& \equiv h^{n+1} \gamma_{n} y^{(n+1)}(\eta),
\end{aligned}
$$

where $\eta$ is an intermediate point in the interval $\left[t_{k-n}, t_{k}\right]$.

In particular, from (3) for a given $n$, we get the methods:

- $n=1$ (Euler's method)

$$
y_{k}=y_{k-1}+h f\left(t_{k-1}, y_{k-1}\right),
$$

$-n=2$

$$
y_{k}=y_{k-1}+\frac{h}{2}\left[3 f\left(t_{k-1}, y_{k-1}\right)-f\left(t_{k-2}, y_{k-2}\right)\right] \text {, }
$$

$-n=3$

$$
y_{k}=y_{k-1}+\frac{h}{12}\left[23 f\left(t_{k-1}, y_{k-1}\right)-16 f\left(t_{k-2}, y_{k-2}\right)+5 f\left(t_{k-3}, y_{k-3}\right)\right],
$$

$-n=4$

$$
\begin{aligned}
y_{k}= & y_{k-1}+\frac{h}{24}\left[55 f\left(t_{k-1}, y_{k-1}\right)-59 f\left(t_{k-2}, y_{k-2}\right)\right. \\
& \left.+37 f\left(t_{k-3}, y_{k-3}\right)-9 f\left(t_{k-4}, y_{k-4}\right)\right] .
\end{aligned}
$$

If in (2) we take the polynomial $\bar{P}(\tau)$ of the degree $n$ (instead of $P(\tau)$ of the degree $n-1$ ) such that

$$
\bar{P}\left(t_{k-j}\right)=f\left(t_{k-j}, y\left(t_{k-j}\right)\right), \quad j=0,1, \ldots, n,
$$

we obtain the $n$-step implicit Adams-Moulton method of the form

$$
y_{k}=y_{k-1}+h \sum_{j=0}^{n} \bar{\gamma}_{j} \nabla^{j} f\left(t_{k}, y_{k}\right)
$$

where

$$
\begin{gathered}
\nabla^{j} f\left(t_{k}, y_{k}\right)=\sum_{m=0}^{j}(-1)^{m}\left(\begin{array}{c}
j \\
m
\end{array}\right) f\left(t_{k-m}, y_{k-m}\right), \\
\bar{\gamma}_{0}=1, \quad \bar{\gamma}_{j}=\frac{1}{j !} \int_{-1}^{0} s(s+1) \cdots(s+j-1) d s \text { for } j=1,2, \ldots, n+1,
\end{gathered}
$$


with truncation error

$$
\begin{aligned}
h^{n+2} \bar{\gamma}_{n+1} \bar{\psi}(\bar{\eta}, y(\bar{\eta})) & \equiv h^{n+2} \bar{\gamma}_{n+1} f^{(n+1)}(\bar{\eta}, y(\bar{\eta})) \\
& \equiv h^{n+2} \bar{\gamma}_{n+1} y^{(n+2)}(\bar{\eta}),
\end{aligned}
$$

where $\bar{\eta}$ is an intermediate point in the interval $\left[t_{k-n}, t_{k}\right]$.

From (11), we have:

- $n=1$ (the trapezoidal rule)

$$
y_{k}=y_{k-1}+\frac{h}{2}\left[f\left(t_{k}, y_{k}\right)+f\left(t_{k-1}, y_{k-1}\right)\right] \text {, }
$$

$-\quad n=2$

$$
y_{k}=y_{k-1}+\frac{h}{12}\left[5 f\left(t_{k}, y_{k}\right)+8 f\left(t_{k-1}, y_{k-1}\right)-f\left(t_{k-2}, y_{k-2}\right)\right],
$$

$-n=3$

$$
\begin{aligned}
y_{k}= & y_{k-1}+\frac{h}{24}\left[9 f\left(t_{k}, y_{k}\right)+19 f\left(t_{k-1}, y_{k-1}\right)\right. \\
& \left.-5 f\left(t_{k-2}, y_{k-2}\right)+f\left(t_{k-3}, y_{k-3}\right)\right] .
\end{aligned}
$$

The initial value problem (1) can be written in another equivalent integral form, namely

$$
y(t)=y\left(t_{k-2}\right)+\int_{t_{k-2}}^{t} f(\tau, y(\tau)) d \tau, \quad t>t_{k-2},
$$

from which we get

$$
y\left(t_{k}\right)=y\left(t_{k-2}\right)+\int_{t_{k-2}}^{t_{k}} f(\tau, y(\tau)) d \tau .
$$

If we approximate the integral in (18) by the polynomial $P(\tau)$ (the same polynomial as before), then we obtain the conventional $n$-step explicit method of Nyström of the form

$$
y_{k}=y_{k-2}+h \sum_{j=0}^{n-1} v_{j} \nabla^{j} f\left(t_{k-1}, y_{k-1}\right)
$$

with the truncation error

$$
h^{n+1}\left[v_{n}^{*} \psi\left(\eta^{*}, y\left(\eta^{*}\right)\right)+v_{n}^{* *} \psi\left(\eta^{* *}, y\left(\eta^{* *}\right)\right)\right],
$$


where $\psi(\eta, y(\eta)) \equiv f^{(n)}(\eta, y(\eta)) \equiv y^{(n+1)}(\eta), \eta^{*}$, and $\eta^{* *}$ are intermediate points in the interval $\left[t_{k-n}, t_{k}\right]$, and

$$
\begin{aligned}
v_{0}=2, \quad v_{j} & =\frac{1}{j !} \int_{-1}^{1} s(s+1) \cdots(s+j-1) d s, j=1,2, \ldots, n-1, \\
v_{n}^{*} & =\frac{1}{n !} \int_{-1}^{0} s(s+1) \cdots(s+n-1) d s, \\
v_{n}^{* *} & =\frac{1}{n !} \int_{0}^{1} s(s+1) \cdots(s+n-1) d s .
\end{aligned}
$$

The coefficients $v_{n}^{*}$ and $v_{n}^{* *}$ are very important in the interval methods considered (see Section 4).

In particular, from (19) for a given $n$, we get:

$-\quad n=1,2$ (the midpoint rule)

$$
y_{k}=y_{k-2}+2 h f\left(t_{k-1}, y_{k-1}\right)
$$

- $n=3$

$$
y_{k}=y_{k-2}+\frac{h}{3}\left[7 f\left(t_{k-1}, y_{k-1}\right)-2 f\left(t_{k-2}, y_{k-2}\right)+f\left(t_{k-3}, y_{k-3}\right)\right],
$$

$-n=4$

$$
\begin{array}{r}
y_{k}=y_{k-2}+\frac{h}{3}\left[8 f\left(t_{k-1}, y_{k-1}\right)-5 f\left(t_{k-2}, y_{k-2}\right)\right. \\
\left.+4 f\left(t_{k-3}, y_{k-3}\right)-f\left(t_{k-4}, y_{k-4}\right)\right] .
\end{array}
$$

If, in (18), we use the polynomial $\bar{P}(\tau)$ (see earlier), we obtain the method

$$
y_{k}=y_{k-2}+h \sum_{j=0}^{n} \bar{v}_{j} \nabla^{j} f\left(t_{k}, y_{k}\right) \text {. }
$$

This is the well-known conventional $n$-step implicit method of Milne-Simpson with the truncation error

$$
h^{n+2}\left[\bar{v}_{n+1}^{*} \bar{\psi}\left(\bar{\eta}^{*}, y\left(\bar{\eta}^{*}\right)\right)+\bar{v}_{n+1}^{* *} \bar{\psi}\left(\bar{\eta}^{* *}, y\left(\bar{\eta}^{* *}\right)\right)\right] \text {, }
$$


where $\bar{\psi}(\bar{\eta}, y(\bar{\eta})) \equiv f^{(n+1)}(\bar{\eta}, y(\bar{\eta})) \equiv y^{(n+2)}(\bar{\eta}), \bar{\eta}^{*}$, and $\bar{\eta}^{* *}$ are some points in the interval $\left[t_{n-k}, t_{k}\right]$,

$$
\begin{aligned}
\bar{v}_{0}=2, \quad \bar{v}_{j} & =\frac{1}{j !} \int_{-2}^{0} s(s+1) \cdots(s+j-1) d s, \quad j=1,2, \ldots, n, \\
\bar{v}_{n+1}^{*} & =\frac{1}{(n+1) !} \int_{-1}^{0} s(s+1) \cdots(s+n) d s \\
\bar{v}_{n+1}^{* *} & =\frac{1}{(n+1) !} \int_{-2}^{-1} s(s+1) \cdots(s+n) d s .
\end{aligned}
$$

As for the method (19), for interval equivalents of (25), it is important to write two coefficients $\bar{v}_{n+1}^{*}$ and $\bar{v}_{n}^{* *}$ instead of one $\bar{v}_{n+1}=\bar{v}_{n+1}^{*}+\bar{v}_{n+1}^{* *}$ (see Section 4 for details).

From (25), for a given $n$, we get the following methods:

- $\quad n=1$ (the midpoint rule)

$$
y_{k}=y_{k-2}+2 h f\left(t_{k-1}, y_{k-1}\right),
$$

- $\quad n=2,3$ (the Milne method)

$$
y_{k}=y_{k-2}+\frac{h}{3}\left[f\left(t_{k}, y_{k}\right)+4 f\left(t_{k-1}, y_{k-1}\right)+f\left(t_{k-2}, y_{k-2}\right)\right] .
$$

If we compare the truncation errors (6) with (14) and (20) with (26), we see that with the same number of steps the order of implicit methods are greater. Unfortunately, in implicit methods, we have to use some iterative process to find $y_{k}$ with a given accuracy. For the initial approximation $y_{k}^{(0)}$ in such a process, one can take $y_{k-1}$, but a better approach is to take for this approximation the value of $y_{k}$ obtained from an explicit method (which usually significantly decreases the number of iterations for a given accuracy). It is well-known that such a combination yields a predictor-corrector method, in which an explicit method is the predictor, and an implicit one fulfills the role of corrector.

\section{Interval versions of explicit multistep methods}

Let us denote:

- $\Delta_{t}$ and $\Delta_{y}$ are the bounded sets in which the function $f(t, y)$, occurring in (1), is defined, i.e.,

$$
\begin{gathered}
\Delta_{t}=\{t \in \mathbb{R}: 0 \leq t \leq a\}, \\
\Delta_{y}=\left\{y=\left(y_{1}, y_{2}, \ldots, y_{N}\right)^{\mathrm{T}} \in \mathbb{R}^{N}: \underline{b}_{i} \leq y_{i} \leq \bar{b}_{i}, i=1,2, \ldots, N\right\},
\end{gathered}
$$


- $\quad F(T, Y)$ is an interval extension of $f(t, y)$, where an interval extension of the function

$$
f: \mathbb{R} \times \mathbb{R}^{N} \supset \Delta_{t} \times \Delta_{y} \rightarrow \mathbb{R}^{N}
$$

we call a function

$$
F: \mathbb{I R} \times \mathbb{I} \mathbb{R}^{N} \supset \mathbb{I} \Delta_{t} \times \mathbb{I} \Delta_{y} \rightarrow \mathbb{I R}^{N}
$$

such that

$$
(t, y) \in(T, Y) \Rightarrow f(t, y) \in F(T, Y),
$$

and where $\mathbb{I}$ and $\mathbb{R}^{N}$ denote the space of real intervals, and the space of $N$ dimensional real interval vectors, respectively,

- $\Psi(T, Y)$ - an interval extension of $\psi(t, y)$ (see (6)),

and let us assume that:

- the function $F(T, Y)$ is defined and continuous for all $T \subset \Delta_{t}$ and $Y \subset \Delta_{y},{ }^{1}$

- the function $F(T, Y)$ is monotonic with respect to inclusion, i.e.,

$$
T_{1} \subset T_{2} \wedge Y_{1} \subset Y_{2} \Rightarrow F\left(T_{1}, Y_{1}\right) \subset F\left(T_{2}, Y_{2}\right),
$$

- $\quad$ for each $T \subset \Delta_{t}$ and for each $Y \subset \Delta_{y}$ there exists a constant $\Lambda>0$ such that

$$
w(F(T, Y)) \leq \Lambda(w(T)+w(Y)),
$$

where $w(A)$ denotes the width of the interval $A$ (if $A=\left(A_{1}, A_{2}, \ldots, A_{N}\right)^{\mathrm{T}}$, then the number $w(A)$ is defined by $\left.w(A)=\max _{i=1,2, \ldots, N} w\left(A_{i}\right)\right)$,

- the function $\Psi(T, Y)$ is defined for all $T \subset \Delta_{t}$ and $Y \subset \Delta_{y}$,

- the function $\Psi(T, Y)$ is monotonic with respect to inclusion.

Moreover, let us assume that $y(0) \in Y_{0}$, and the intervals $Y_{k}$ such that $y\left(t_{k}\right) \in Y_{k}$ for $k=1,2, \ldots, n-1$ are known. We can obtain such $Y_{k}$ by applying an interval one-step method, for example, an interval method of Runge-Kutta (see, e.g., [28, 41]) or interval methods based on the Taylor series (see, e.g., [2-4, 7, 13, 20, 34, 36]). The first approach to construct an interval version of Adams-Bashforth method has been made by Yu. I. Shokin [41]. Unfortunately, as we have shown in [19] and [28], his formula fails even in the simplest case, i.e., when $n=1$. Correcting a defective error term in Shokin's formula, we obtain the correct formula for interval methods of Adams-Bashforth type of the following form:

$$
\begin{aligned}
Y_{k}= & Y_{k-1}+h \sum_{j=0}^{n-1} \gamma_{j} \nabla^{j} F_{k-1} \\
+ & h^{n+1} \gamma_{n} \Psi\left(T_{k-1}+[-(n-1) h, h], Y_{k-1}+[-(n-1) h, h] F\left(\Delta_{t}, \Delta_{y}\right)\right) \\
& k=n, n+1, \ldots, m,
\end{aligned}
$$

\footnotetext{
${ }^{1}$ The function $F(T, Y)$ is continuous at $\left(T_{0}, Y_{0}\right)$ if for every $\varepsilon>0$ there is a positive number $\delta=\delta(\varepsilon)$ such that $d\left(F(T, Y), F\left(T_{0}, Y_{0}\right)\right)<\varepsilon$ whenever $d\left(T, T_{0}\right)<\delta$ and $d\left(Y, Y_{0}\right)<\delta$. If $Y$ is an interval vector, the second inequality should be fulfilled for each component. Here, $d$ denotes the interval metric defined by $d\left(X_{1}, X_{2}\right)=\max \left\{\left|\underline{X}_{1}-\underline{X}_{2}\right|,\left|\bar{X}_{1}-\bar{X}_{2}\right|\right\}$, where $X_{1}=\left[\underline{X}_{1}, \bar{X}_{1}\right]$ and $X_{2}=\left[\underline{X}_{2}, \bar{X}_{2}\right]$ are two intervals.
} 
where

$$
F_{k-1}=F\left(T_{k-1}, Y_{k-1}\right), \quad h=\frac{a}{m}, \quad t_{k}=k h \in T_{k}, \quad k=0,1, \ldots, m,
$$

$a<\infty$ is a constant occurring in the definition of $\Delta_{t}$, the coefficients $\gamma_{j}$, where $j=0,1, \ldots, n$, are given by $(5)$, and $[-(n-1) h, h]$ means the (closed) interval with the left border $-(n-1) h$ and the right border $h$, respectively.

Since

$$
\nabla^{j} F_{k-1}=\sum_{m=0}^{j}(-1)^{m}\left(\begin{array}{c}
j \\
m
\end{array}\right) F_{k-1-m},
$$

the formula (30) can be written in the equivalent form

$$
\begin{aligned}
Y_{k}= & Y_{k-1}+h \sum_{j=1}^{n} \beta_{n j} F_{k-j} \\
& +h^{n+1} \gamma_{n} \Psi\left(T_{k-1}+[-(n-1) h, h], Y_{k-1}+[-(n-1) h, h] F\left(\Delta_{t}, \Delta_{y}\right)\right),
\end{aligned}
$$

where the coefficients $\beta_{n j}$ are given by

$$
\beta_{n j}=(-1)^{j-1} \sum_{m=j-1}^{n-1}\left(\begin{array}{c}
m \\
j-1
\end{array}\right) \gamma_{m}, \quad j=1,2, \ldots, n .
$$

In particular, for a given $n$, we get (from (30) and (32)) the following methods:

- $\quad n=1$ (the interval version of Euler's method (7))

$$
Y_{k}=Y_{k-1}+h F\left(T_{k-1}, Y_{k-1}\right)+\frac{h^{2}}{2} \Psi\left(T_{k-1}+[0, h], Y_{k-1}+[0, h] F\left(\Delta_{t}, \Delta_{y}\right)\right),
$$

- $\quad n=2$ (the interval version of the method (8))

$$
\begin{aligned}
Y_{k}= & Y_{k-1}+\frac{h}{2}\left(3 F\left(T_{k-1}, Y_{k-1}\right)-F\left(T_{k-2}, Y_{k-2}\right)\right) \\
& +\frac{5 h^{3}}{12} \Psi\left(T_{k-1}+[-h, h], Y_{k-1}+[-h, h] F\left(\Delta_{t}, \Delta_{y}\right)\right),
\end{aligned}
$$

$-\quad n=3$ (the interval version of the method (9))

$$
\begin{aligned}
Y_{k}= & Y_{k-1}+\frac{h}{2}\left(23 F\left(T_{k-1}, Y_{k-1}\right)-16 F\left(T_{k-2}, Y_{k-2}\right)+5 F\left(T_{k-3}, Y_{k-3}\right)\right) \\
& +\frac{3 h^{4}}{8} \Psi\left(T_{k-1}+[-2 h, h], Y_{k-1}+[-2 h, h] F\left(\Delta_{t}, \Delta_{y}\right)\right),
\end{aligned}
$$

$-\quad n=4$ (the interval version of the method (10))

$$
\begin{aligned}
Y_{k}= & Y_{k-1}+\frac{h}{24}\left(55 F\left(T_{k-1}, Y_{k-1}\right)-59 F\left(T_{k-2}, Y_{k-2}\right)\right. \\
& \left.+37 F\left(T_{k-3}, Y_{k-3}\right)-9 F\left(T_{k-4}, Y_{k-4}\right)\right) \\
& +\frac{251 h^{5}}{720} \Psi\left(T_{k-1}+[-3 h, h], Y_{k-1}+[-3 h, h] F\left(\Delta_{t}, \Delta_{y}\right)\right) .
\end{aligned}
$$


Assuming that $F(T, Y)$ and $\Psi(T, Y)$ fulfill the same conditions as previously, the explicit interval methods of Nyström type, we define as follows:

$$
\begin{gathered}
Y_{k}=Y_{k-2}+h \sum_{j=0}^{n-1} \gamma_{j} \nabla^{j} F_{k-1}+h^{n+1}\left(v_{n}^{*} \Psi_{n}+v_{n}^{* *} \Psi_{n}\right), \\
k=n, n+1, \ldots, m,
\end{gathered}
$$

where

$$
\Psi_{n}=\Psi\left(T_{k-1}+[-(n-1) h, h], Y_{k-1}+[-(n-1) h, h] F\left(\Delta_{t}, \Delta_{y}\right)\right),
$$

and $\Psi(T, Y)$ is an interval extension of $\psi(t, y(t)) \equiv f^{(n)}(t, y(t)) \equiv y^{(n+1)}(t)$.

Let us note that in (37), we cannot write $\left(v_{n}^{*}+v_{n}^{* *}\right) \Psi_{n}$ instead of $v_{n}^{*} \Psi_{n}++v_{n}^{* *} \Psi_{n}$, because in general $\left|v_{n}^{*}+v_{n}^{* *}\right|$ may be different from $\left|v_{n}^{*}\right|+\left|v_{n}^{* *}\right|$. Moreover, the formula (37) can be written in more convenient form

$$
Y_{k}=Y_{k-2}+h \sum_{j=1}^{n} \delta_{n j} F_{k-j}+h\left(v_{n}^{*} \Psi_{n}+v_{n}^{* *} \Psi_{n}\right), \quad k=n, n+1, \ldots, m,
$$

where

$$
\delta_{n j}=(-1)^{j-1} \sum_{m=j-1}^{n-1}\left(\begin{array}{c}
m \\
j-1
\end{array}\right) v_{m}, \quad j=1,2, \ldots, n .
$$

In particular, for a given $n$ from (37) and (38), we have the following methods:

$-\quad n=1$ (the interval midpoint rule)

$$
Y_{k}=Y_{k-2}+2 h F\left(T_{k-1}, Y_{k-1}\right)+\frac{h^{2}}{2}\left(\Psi_{1}-\Psi_{1}\right),
$$

where

$$
\Psi_{1}=\Psi\left(T_{k-1}+[0, h], Y_{k-1}+[0, h] F\left(\Delta_{t}, \Delta_{y}\right)\right),
$$

- $n=2$ (in the conventional case we have the same method as for $n=1$ )

$$
Y_{k}=Y_{k-2}+2 h F\left(T_{k-1}, Y_{k-1}\right)+\frac{h^{3}}{12}\left(5 \Psi_{2}-\Psi_{2}\right)
$$

where

$$
\Psi_{2}=\Psi\left(T_{k-1}+[-h, h], Y_{k-1}+[-h, h] F\left(\Delta_{t}, \Delta_{y}\right)\right),
$$

- $n=3$ (the interval version of the method (23))

$$
\begin{aligned}
Y_{k}= & Y_{k-2}+\frac{h}{3}\left(7 F\left(T_{k-1}, Y_{k-1}\right)-2 F\left(T_{k-2}, Y_{k-2}\right)+F\left(T_{k-3}, Y_{k-3}\right)\right) \\
& +\frac{h^{4}}{24}\left(9 \Psi_{3}-\Psi_{3}\right)
\end{aligned}
$$

where

$$
\Psi_{3}=\Psi\left(T_{k-1}+[-2 h, h], Y_{k-1}+[-2 h, h] F\left(\Delta_{t}, \Delta_{y}\right)\right),
$$


$-\quad n=4$ (the interval version of the method (24))

$$
\begin{aligned}
Y_{k}= & Y_{k-2}+\frac{h}{3}\left(8 F\left(T_{k-1}, Y_{k-1}\right)-5 F\left(T_{k-2}, Y_{k-2}\right)+4 F\left(T_{k-3}, Y_{k-3}\right)\right. \\
& \left.-F\left(T_{k-4}, Y_{k-4}\right)\right)+\frac{h^{5}}{720}\left(251 \Psi_{4}-19 \Psi_{4}\right)
\end{aligned}
$$

where

$$
\Psi_{4}=\Psi\left(T_{k-1}+[-3 h, h], Y_{k-1}+[-3 h, h] F\left(\Delta_{t}, \Delta_{y}\right)\right) .
$$

The subtractions like these, occurring in (39)-(42), often yield overestimation. But these subtractions are simple consequences of coefficient values in (38).

In $[19,26,28]$, we have proved that for the methods (30) and (37) (or (32) and (38), respectively) we have $y\left(t_{k}\right) \in Y_{k}$, where $y(t)$ is the exact solution of the initial value problem (1), and we have also estimated the widths $w\left(Y_{k}\right)$ of the intervals $Y_{k}$ obtained by these methods.

\section{Interval versions of implicit multistep methods}

As previously, let us denote by $\Delta_{t}$ and $\Delta_{y}$ the sets in which the function $f(t, y)$ is defined, and let $F(T, Y)$ and $\bar{\Psi}(T, Y)$ denote interval extensions of $f(t, y)$ and $\bar{\Psi}(t, y(t))$, respectively, (other assumptions for $F(T, Y)$ and $\bar{\Psi}(T, Y)$ are the same as for $F(T, Y)$ and $\Psi(T, Y)$ in the previous section). Let us also assume that $y(0) \in Y_{0}$, and the intervals $Y_{k}$ such that $y\left(t_{k}\right) \in Y_{k}, k=1,2, \ldots, n-1$ are known. The implicit interval $n$-step methods of Adams-Moulton type can be defined as

$$
\begin{aligned}
& Y_{k}= Y_{k-1}+h \sum_{j=0}^{n} \bar{\gamma}_{j} \nabla^{j} F_{k} \\
&+h^{n+2} \bar{\gamma}_{n+1} \bar{\Psi}\left(T_{k}+[-n h, 0], Y_{k}+[-n h, 0] F\left(\Delta_{t}, \Delta_{y}\right)\right), \\
& \quad k=n, n+1, \ldots, m,
\end{aligned}
$$

where $\overline{\gamma_{j}}, j=0,1, \ldots, n+1$ are given by (13), $F_{k}=F\left(T_{k}, Y_{k}\right)$ and

$$
\nabla^{j} F_{k}=\sum_{m=0}^{j}(-1)^{m}\left(\begin{array}{c}
j \\
m
\end{array}\right) F_{k-m} .
$$

Applying (44), (43) can be written in the equivalent form

$$
\begin{aligned}
Y_{k}= & Y_{k-1}+h \sum_{j=1}^{n} \bar{\gamma}_{j} \sum_{m=1}^{n}(-1)^{m}\left(\begin{array}{c}
j \\
m
\end{array}\right) F_{k-m} \\
& +h^{n+2} \bar{\gamma}_{n+1} \bar{\Psi}\left(T_{k}+[-n h, 0], Y_{k}+[-n h, 0] F\left(\Delta_{t}, \Delta_{y}\right)\right) .
\end{aligned}
$$

It is well-known that in interval arithmetic the distributive law is not generally satisfied. This means that the values of the interval extensions of $f$ in the above formulas with the same indices cannot be subtracted. 
According to (12), in real arithmetic, we have

$$
\sum_{j=0}^{n} \bar{\beta}_{n j} f_{k-j}=\sum_{j=0}^{n} \bar{\gamma}_{j} \nabla^{j} f_{k},
$$

where $f_{k-j}=f\left(t_{k-j}, y\left(t_{k-j}\right)\right), j=0,1, \ldots, n$, and

$$
\bar{\beta}_{n j}=(-1)^{j} \sum_{m=j}^{n}\left(\begin{array}{c}
m \\
j
\end{array}\right) \bar{\gamma}_{m} .
$$

Hence, formula (11) is equivalent to

$$
y_{k}=y_{k-1}+h \sum_{j=0}^{n} \bar{\beta}_{n j} f_{k-j} .
$$

But in interval arithmetic, we have

$$
\sum_{j=0}^{n} \bar{\beta}_{n j} F_{k-j} \subset \sum_{j=0}^{n} \bar{\gamma}_{j} \nabla^{j} F_{k},
$$

where the subset relation $(\subset)$ is defined as not necessarily proper, and we get another kind of implicit interval methods corresponding to the conventional formula (46), namely

$$
\begin{gathered}
Y_{k}=Y_{k-1}+h \bar{\beta}_{n 0} F_{k}+h \sum_{j=1}^{n} \bar{\beta}_{n j} F_{k-j} \\
+h^{n+2} \bar{\gamma}_{n+1} \bar{\Psi}\left(T_{k}+[-n h, 0], Y_{k}+[-n h, 0] F\left(\Delta_{t}, \Delta_{y}\right)\right), \\
k=n, n+1, \ldots, m .
\end{gathered}
$$

For a given $n$ from (48), we get the following methods:

- $n=1$ (the interval trapezoidal rule)

$$
\begin{aligned}
Y_{k}= & Y_{k-1}+\frac{h}{2}\left(F\left(T_{k}, Y_{k}\right)+F\left(T_{k-1}, Y_{k-1}\right)\right) \\
& -\frac{h^{3}}{12} \Psi\left(T_{k}+[-h, 0], Y_{k}+[-h, 0] F\left(\Delta_{t}, \Delta_{y}\right)\right),
\end{aligned}
$$

$-\quad n=2$ (the interval version of the method (16))

$$
\begin{aligned}
Y_{k}= & \left.Y_{k-1}+\frac{h}{12}\left(5 F\left(T_{k}, Y_{k}\right)+8 F\left(T_{k-1}, Y_{k-1}\right)\right)-F\left(T_{k-2}, Y_{k-2}\right)\right) \\
& -\frac{h^{4}}{24} \bar{\Psi}\left(T_{k}+[-2 h, 0], Y_{k}+[-2 h, 0] F\left(\Delta_{t}, \Delta_{y}\right)\right),
\end{aligned}
$$


$-\quad n=3$ (the interval version of the method (17))

$$
\begin{aligned}
Y_{k}= & Y_{k-1}+\frac{h}{24}\left(9 F\left(T_{k}, Y_{k}\right)+19 F\left(T_{k-1}, Y_{k-1}\right)\right. \\
& \left.\left.-5 F\left(T_{k-2}, Y_{k-2}\right)\right)+F\left(T_{k-3}, Y_{k-3}\right)\right) \\
& -\frac{19 h^{5}}{720} \bar{\Psi}\left(T_{k}+[-3 h, 0], Y_{k}+[-3 h, 0] F\left(\Delta_{t}, \Delta_{y}\right)\right) .
\end{aligned}
$$

If we denote by $Y_{k}^{1}$ the interval solutions obtained from the formula (43) (or (45)), i.e. from the formula with backward interval differences, and by $Y_{k}^{2}$ the interval solutions obtained from (48), then from (47), we have immediately that $Y_{k}^{2} \subset Y_{k}^{1}$. It means that the second kind of implicit interval formula gives the interval solution with a smaller width, i.e., it is better. Thus, we should rather apply the methods (49)-(51) instead of the methods obtained for the same $n$ from (45).

Let us note that (43) (or (45)) and (48) are nonlinear interval equations with respect to $Y_{k}, k=n, n+1, \ldots, m$. This implies that in each step of implicit interval methods we have to solve an interval equation of the form

$$
X=G(T, X),
$$

where

$$
T \in \mathbb{I} \Delta_{t} \subset \mathbb{I} \mathbb{R}, X=\left(X_{1}, X_{2}, \ldots, X_{N}\right)^{T} \in \mathbb{I} \Delta_{y} \subset \mathbb{I} \mathbb{R}^{N}, G: \mathbb{I} \Delta_{t} \times \mathbb{I} \Delta_{y} \rightarrow \mathbb{I}^{N} \text {. }
$$

If $G$ is a contraction mapping, ${ }^{2}$ then using the well-known fixed-point theorem, we can apply the iteration

$$
X^{(l)}=G\left(T, X^{(l)}\right), \quad l=0,1, \ldots,
$$

which is convergent to $X^{*}$, i.e., $\quad \lim _{l \rightarrow \infty} X^{l}=X^{*}$, for an arbitrary choice of $X^{(0)} \in \mathbb{I} \Delta_{y}$.

For the interval methods of Adams-Moulton type given by (48), the iteration (52) is of the form

$$
\begin{aligned}
& Y_{k}^{(l+1)}= Y_{k-1}+h \bar{\beta}_{n 0} F\left(T_{k}, Y_{k}^{(l)}\right)+h \sum_{j=1}^{n} \bar{\beta}_{n j} F\left(T_{k-j}, Y_{k-j}\right) \\
&+h^{n+2} \bar{\gamma}_{n+1} \bar{\Psi}\left(T_{k}+[-n h, 0], Y_{k}^{(l)}+[-n h, 0] F\left(\Delta_{t}, \Delta_{y}\right)\right), \\
& l=0,1, \ldots, \quad k=n, n+1, \ldots, m .
\end{aligned}
$$

On the basis of (25) and (26), we can define the implicit interval methods of the form $[26,28]$ :

$$
\begin{gathered}
\left.Y_{k}=Y_{k-2}+h \sum_{j=0}^{n} \bar{v}_{j} \nabla^{j} F_{k}+h^{n+2}\left(\bar{v}_{n+1}^{*} \bar{\Psi}_{n}\right)+\bar{v}_{n+1}^{* *} \bar{\Psi}_{n}\right) \\
k=n, n+1, \ldots, m,
\end{gathered}
$$

\footnotetext{
${ }^{2} \mathrm{~A}$ map $G$ is a contraction mapping if there exists a constant $q$, with $0 \leq q<1$, such that $d(G(T, X), G(T, Y)) \leq q d(X, Y)$ for all $X, Y \in \mathbb{I} \Delta_{y}$. Here, again, $d$ is the interval metric.
} 
where $F_{k}=F\left(T_{k}, Y_{k}\right)$ and where

$$
\bar{\Psi}_{n}=\bar{\Psi}\left(T_{k}+[-n h, 0], Y_{k}+[-n h, 0] F\left(\Delta_{t}, \Delta_{y}\right)\right) .
$$

Because of

where

$$
\sum_{j=0}^{n} \bar{v}_{j} \nabla^{j} f_{k}=\sum_{j=0}^{n} \bar{\delta}_{n j} f_{k-j} .
$$

$$
\bar{\delta}_{n j}=(-1)^{j} \sum_{m=j}^{n}\left(\begin{array}{c}
m \\
j
\end{array}\right) \bar{v}_{m},
$$

we can write the second kind of interval methods of Milne-Simpson type as follows $[26,28]$ :

$$
\begin{gathered}
Y_{k}=Y_{k-2}+h \sum_{j=0}^{n} \bar{\delta}_{n j} F_{k-j}+h^{n+2}\left(\bar{v}_{n+1}^{*} \bar{\Psi}_{n}+\bar{v}_{n+1}^{* *} \bar{\Psi}_{n}\right), \\
k=n, n+1, \ldots, m .
\end{gathered}
$$

Below are examples of implicit methods of the second kind (obtained from (55)).

$-n=1$

where

$$
Y_{k}=Y_{k-2}+2 h F\left(T_{k-1}, Y_{k-1}\right)+\frac{h^{3}}{12}\left(5 \bar{\Psi}_{1}-\bar{\Psi}_{1}\right),
$$

$$
\bar{\Psi}_{1}=\bar{\Psi}\left(T_{k}+[-h, 0], Y_{k}+[-h, 0] F\left(\Delta_{t}, \Delta_{y}\right)\right),
$$

- $\quad n=2$ (the interval version of Milne's method (29))

$$
\begin{aligned}
Y_{k}= & Y_{k-2}+\frac{h}{3}\left(F\left(T_{k}, Y_{k}\right)+4 F\left(T_{k-1}, Y_{k-1}\right)\right. \\
& \left.+F\left(T_{k-2}, Y_{k-2}\right)\right)+\frac{h^{4}}{24}\left(\bar{\Psi}_{2}-\bar{\Psi}_{2}\right),
\end{aligned}
$$

where

$$
\bar{\Psi}_{2}=\bar{\Psi}\left(T_{k}+[-2 h, 0], Y_{k}+[-2 h, 0] F\left(\Delta_{t}, \Delta_{y}\right)\right),
$$

$-n=3$

where

$$
\begin{aligned}
Y_{k}= & Y_{k-2}+\frac{h}{3}\left(F\left(T_{k}, Y_{k}\right)+4 F\left(T_{k-1}, Y_{k-1}\right)\right. \\
& \left.+F\left(T_{k-2}, Y_{k-2}\right)\right)+\frac{h^{5}}{720}\left(11 \bar{\Psi}_{3}-19 \bar{\Psi}_{3}\right),
\end{aligned}
$$

As previously, the subtractions in (56)-(58) are consequences of coefficient values (in (55) at present).

Denoting by $Y_{k}^{1}$ the interval solution obtained from (54) and by $Y_{k}^{2}$ the one obtained from (55), we have the same inclusion as in the case of interval methods of Adams-Moulton type, i.e., $Y_{k}^{2} \subset Y_{k}^{1}$. It follows immediately from the fact that

$$
\sum_{j=0}^{n} \bar{\delta}_{n j} F_{k-j} \subset \sum_{j=0}^{n} \bar{v}_{j} \nabla^{j} F_{k} .
$$


Thus, one should use the methods (56)-(58) instead of the methods obtained for the same $n$ from (54).

In each step of the interval methods of Milne-Simpson type (of both kinds), we have to solve a system of nonlinear interval equations. If the right-hand sides of (54) and (55) are contracting mappings, then the iterations follows immediately from the fixed-point theorem. For the second kind of methods, i.e., for (55), the iteration is

$$
\begin{aligned}
Y_{k}^{(l+1)}= & Y_{k-2}+h \bar{\delta}_{n 0} F\left(T_{k}, Y_{k}^{(l)}\right)+h \sum_{j=1}^{n} \bar{\delta}_{n j} F\left(T_{k-j}, Y_{k-j}\right) \\
+ & h^{n+2}\left(\bar{v}_{n+1}^{*} \bar{\Psi}_{n}^{(l)}+\bar{v}_{n+1}^{* *} \bar{\Psi}_{n}^{(l)}\right), \\
& l=0,1, \ldots, \quad k=n, n+1, \ldots, m,
\end{aligned}
$$

where

$$
\bar{\Psi}_{n}^{(l)}=\bar{\Psi}\left(T_{k}+[-n h, 0], Y_{k}^{(l)}+[-n h, 0] F\left(\Delta_{t}, \Delta_{y}\right)\right) .
$$

In $[18,20,26,28]$, we have proved that the exact solution of the initial value problem (1) belongs to the interval solutions $Y_{k}$ obtained by the implicit interval multistep methods considered in this section. In the same papers, we have estimated the widths of these solutions.

\section{Interval predictor-corrector methods}

In each multistep interval method, both the explicit ones presented in Section 3 and the implicit ones given in Section 4, we need initial intervals. For any interval $n$-step method, we have to know the intervals $Y_{k}$ for $k=0,1, \ldots, n-1$ such that $y\left(t_{k}\right) \in Y_{k}$, where $y(t)$ denotes the exact solution of the initial value problem (1). The interval $Y_{0}$ can be determined easy from the initial condition, but to obtain the remaining intervals, we have to use some one-step method, for example, the interval methods of Runge-Kutta type (see, e.g., [28, 41]), Shokin's method based on the Simpson formula [41], Moore's method [32,33] or the methods based on the high-order Taylor series (see, e.g., [2-4, 7, 17, 22, 36, 38, 40]).

Using explicit interval multistep methods, for given $Y_{k}, k=0,1, \ldots, n-1$, we simply get $Y_{k}$ for $k=n, n+1, \ldots, m$. In implicit interval multistep methods in each step $k=n, n+1, \ldots, m$, we need an initial approximation $Y_{k}^{(0)}$ to start the iteration. One can use $Y_{k}^{(0)}=Y_{k-1}$, but a better approach (as in conventional case) is to take for the initial approximation the solution $Y_{k}$ obtained from any of explicit interval method. This leads to interval predictor-corrector methods. As it is shown in the next section, such an approach significantly reduces the number of iterations needed in implicit methods to obtain interval solutions with the same accuracy (in comparison to the case when in a corrector we take $Y_{k}^{(0)}=Y_{k-1}$ ).

Although as an interval predictor for an $n$-step interval corrector, we can take any explicit method with the number of steps not greater than $n$, we recommend to use a predictor and corrector with the same $n$. In this case, when the interval predictor yields an initial approximation of order $p$, then from the interval corrector we obtained an interval solution of order $p+1$. But we do not recommend to use 
interval predictor-corrector methods for large $n$ (see a note in Example 1 in the next section), and our proposal is to use in practice interval predictor-corrector methods presented in Table 1.

\section{Numerical examples}

In this section, we present a number of examples obtained from an implementation of floating-point interval arithmetic in Delphi Pascal, one of the most popular programming language developed by Borland Software Corporation (Embarcadero Technologies, Inc. at present). This implementation has been written in the form of a unit called IntervalArithmetic32and64 (the current version of this unit is presented in [30]). This unit takes advantage of the Delphi Pascal floating-point Extended type and makes it possible to:

- represent any input numerical data in the form of a machine interval (for a real number the ends of this interval are equal or are two subsequent machine numbers),

- perform all calculations in floating-point interval arithmetic,

- use some standard interval functions,

- give results in the form of proper intervals (if the ends of an interval are not the same machine numbers, one can see the difference in the output).

All programs written in Delphi Pascal for the examples presented can be found in [29]. In [29], it is also included a Delphi Pascal program for solving any initial value problem by all explicit, implicit, and predictor-corrector interval methods considered in this paper. This program requires the user to write a dynamic link library with definitions of appropriate interval functions and to determine starting intervals.

At first, let us consider the commonly used test problem

$$
y^{\prime}=\lambda y, \quad y(0)=1
$$

This problem has the exact solution of the form $y=\exp (\lambda t)$. For $\lambda=0.5$ and $t=0.2,0.6,1.0$, the numerical values of the solution are given in Table 2 .

Since for the problem (60), the exact solution is known, the starting intervals for interval multistep methods can be obtained directly from this solution. But in general, we need an one-step method to determine these intervals, and we use the interval

Table 1 Proposals of interval predictor-corrector methods

\begin{tabular}{llll}
\hline$n$ & Method & Predictor & Corrector \\
\hline 1 & ABM1 & $(33)$ & $(49)$ \\
& NMS1 & $(39)$ & $(56)$ \\
2 & ABM2 & $(34)$ & $(50)$ \\
& NMS2 & $(40)$ & $(57)$ \\
3 & ABM3 & $(35)$ & $(51)$ \\
& NMS3 & $(41)$ & $(58)$ \\
\hline
\end{tabular}


Table 2 The approximate (with 15 digits after decimal point) exact solution to (60) with $\lambda=0.5$

\begin{tabular}{ll}
\hline$t$ & $y(t)$ \\
\hline 0.2 & 1.105170918075648 \\
0.6 & 1.348858807576003 \\
1.0 & 1.648721270700128 \\
\hline
\end{tabular}

version of a Runge-Kutta method (of the fourth order-see [28] or [41]). The starting intervals for the problem (60) and interval multistep methods considered are presented in Table 3, where $\underline{x}$ denotes the largest machine number less or equal to $x$ (similarly, $\bar{x}$ denotes the smallest machine number greater or equal to $x$ ).

Example 1 For the problem (60), and $h=0.0005$ in Table 4, we present the results obtained by explicit interval methods of Adams-Bashforth type for different number of steps $n$, while the results obtained by explicit interval methods of Nyström type are presented in Table 5. It is interesting that for the problem considered and for the same $n$ interval methods of Nyström type give interval solutions with smaller widths. We can also observe that in each case the exact solution belongs to the interval solution obtained (compare Table 2). Moreover, it can be seen that increasing $n$ we obtain better solutions taking into account the widths of intervals. But that is true only for $n \leq 4$. If we use the interval methods of Adams-Bashforth type and Nyström type for $n>4$, then we obtain intervals with greater widths. This is caused by a great number of calculations in these methods and by significant increase of rounding errors following from that, which is not compensated for the method orders. We can generalize this note: the number of steps $n$ and the step size $h$ should be suitably selected for each problem considered.

Example 2 For implicit interval methods of Adams-Moulton and Milne-Simpson types, we have used iterations given by (53) and (59), respectively. The iterations have been stopped when for the interval $Y_{k}^{(l+1)}=\left[\underline{Y}_{k}^{(l+1)}, \bar{Y}_{k}^{(l+1)}\right]$ the following inequalities have been fulfilled:

$$
\left|\frac{\underline{Y}_{k}^{(l+1)}-\underline{Y}_{k}^{(l)}}{\underline{Y}_{k}^{(l)}}\right|<\epsilon,
$$

Table 3 Starting intervals for interval multistep methods and the problem (60)

\begin{tabular}{lll}
\hline$k$ & $t=k h \in T_{k}$ & $Y_{k}$ \\
\hline 1 & 0.0005 & {$[\underline{1.0002500312526043}, \overline{1.0002500312526044}]$} \\
2 & 0.0010 & {$[\underline{1.0005001250208359}, \overline{1.0005001250208360}]$} \\
3 & 0.0015 & {$[\underline{1.0007502813203256}, \overline{1.0007502813203257}]$}
\end{tabular}


Table 4 The solutions of (60) obtained by interval methods of Adams-Bashforth type

\begin{tabular}{|c|c|c|c|}
\hline Method & $t=k h \in T_{k}$ & $Y_{k}$ & Width \\
\hline \multirow{4}{*}{$\begin{array}{l}(33) \\
n=1\end{array}$} & 0.2 & [ $1.1051709169246437 \mathrm{E}+0000$, & $\approx 5.42 \times 10^{-9}$ \\
\hline & & $1.1051709223468415 \mathrm{E}+0000]$ & \\
\hline & 0.6 & $\begin{array}{r}{[1.3498588033584851 \mathrm{E}+0000} \\
1.3498588213958252 \mathrm{E}+0000]\end{array}$ & $\approx 1.80 \times 10^{-8}$ \\
\hline & 1.0 & $\begin{array}{r}1.6487212621146479 \mathrm{E}+0000 \\
1.6487212955601577 \mathrm{E}+0000]\end{array}$ & $\approx 3.34 \times 10^{-8}$ \\
\hline \multirow[t]{3}{*}{$\begin{array}{l}(34) \\
n=2\end{array}$} & 0.2 & $\begin{array}{r}{[1.1051709180745339 \mathrm{E}+0000} \\
1.1051709180769049 \mathrm{E}+0000]\end{array}$ & $\approx 2.37 \times 10^{-12}$ \\
\hline & 0.6 & $\begin{array}{r}{\left[\begin{array}{l}1.3498588075718577 \mathrm{E}+0000 \\
1.3498588075806753 \mathrm{E}+0000]\end{array}\right.}\end{array}$ & $\approx 8.82 \times 10^{-12}$ \\
\hline & 1.0 & $\begin{array}{r}{[1.6487212706914478 \mathrm{E}+0000} \\
1.6487212707098811 \mathrm{E}+0000]\end{array}$ & $\approx 1.84 \times 10^{-11}$ \\
\hline \multirow[t]{3}{*}{$\begin{array}{l}(35) \\
n=3\end{array}$} & 0.2 & $\begin{array}{r}{[1.1051709180756470 \mathrm{E}+0000} \\
1.1051709180756482 \mathrm{E}+0000]\end{array}$ & $\approx 1.07 \times 10^{-15}$ \\
\hline & 0.6 & $\begin{array}{r}{\left[\begin{array}{l}1.3498588075760007 \mathrm{E}+0000 \\
1.3498588075760053 \mathrm{E}+0000]\end{array}\right.}\end{array}$ & $\approx 4.48 \times 10^{-15}$ \\
\hline & 1.0 & $\begin{array}{r}{[1.6487212707001222 \mathrm{E}+0000} \\
1.6487212707001339 \mathrm{E}+0000]\end{array}$ & $\approx 1.15 \times 10^{-14}$ \\
\hline \multirow[t]{3}{*}{$\begin{array}{l}(36) \\
n=4\end{array}$} & 0.2 & $\begin{array}{r}{[1.1051709180756474 \mathrm{E}+0000} \\
1.1051709180756478 \mathrm{E}+0000]\end{array}$ & $\approx 2.55 \times 10^{-16}$ \\
\hline & 0.6 & $\begin{array}{r}{[1.3498588075760025 \mathrm{E}+0000} \\
1.3498588075760037 \mathrm{E}+0000]\end{array}$ & $\approx 1.14 \times 10^{-15}$ \\
\hline & 1.0 & $\begin{array}{r}{[1.6487212707001259 \mathrm{E}+0000} \\
1.6487212707001305 \mathrm{E}+0000]\end{array}$ & $\approx 4.51 \times 10^{-15}$ \\
\hline
\end{tabular}

and

$$
\left|\frac{\bar{Y}_{k}^{(l+1)}-\bar{Y}_{k}^{(l)}}{\bar{Y}_{k}^{(l)}}\right|<\epsilon, \quad \underline{Y}_{k}^{(l)} \neq 0, \quad \bar{Y}_{k}^{(l)} \neq 0, \quad l=0,1, \ldots,
$$

where $\epsilon$ denotes an accuracy given beforehand. Taking $\epsilon=10^{-18}$ for the problem (60), we have obtained results presented in Tables 6 and 7. In each step, the number of iterations has been approximately 5 .

Example 3 For the problem (60), and interval predictor-corrector methods of AdamsBashforth-Moulton and Nyström-Milne-Simpson types, the interval results are the same as for implicit interval methods of Adams-Moulton and Milne-Simpson types, respectively. But using explicit interval methods as predictors, we observe significant 
Table 5 The solutions of (60) obtained by interval methods of Nyström type

\begin{tabular}{|c|c|c|c|}
\hline Method & $t=k h \in T_{k}$ & $Y_{k}$ & Width \\
\hline \multirow{4}{*}{$\begin{array}{l}(39) \\
n=1\end{array}$} & 0.2 & [ $1.1051709142138796 \mathrm{E}+0000$ & $\approx 5.42 \times 10^{-9}$ \\
\hline & & $1.1051709196354981 \mathrm{E}+0000]$ & \\
\hline & 0.6 & $\begin{array}{r}\text { [ } 1.3498587943404760 \mathrm{E}+0000 \\
1.3498588123764994 \mathrm{E}+0000]\end{array}$ & $\approx 1.80 \times 10^{-8}$ \\
\hline & 1.0 & $\begin{array}{r}{[1.6487212453923441 \mathrm{E}+0000} \\
1.6487212788364476 \mathrm{E}+0000]\end{array}$ & $\approx 3.34 \times 10^{-8}$ \\
\hline \multirow{4}{*}{$\begin{array}{l}(40) \\
n=2\end{array}$} & 0.2 & [ $1.1051709180749699 \mathrm{E}+0000$ & $\approx 1.36 \times 10^{-12}$ \\
\hline & & $1.1051709180763254 \mathrm{E}+0000]$ & \\
\hline & 0.6 & $\begin{array}{r}{[1.3498588075737485 \mathrm{E}+0000} \\
1.3498588075782577 \mathrm{E}+0000]\end{array}$ & $\approx 4.51 \times 10^{-12}$ \\
\hline & 1.0 & $\begin{array}{r}{[1.6487212706959476 \mathrm{E}+0000} \\
1.6487212707043086 \mathrm{E}+0000]\end{array}$ & $\approx 8.36 \times 10^{-12}$ \\
\hline \multirow[t]{3}{*}{$\begin{array}{l}(41) \\
n=3\end{array}$} & 0.2 & $\begin{array}{r}{[1.1051709180756473 \mathrm{E}+0000} \\
1.1051709180756480 \mathrm{E}+0000]\end{array}$ & $\approx 5.78 \times 10^{-16}$ \\
\hline & 0.6 & $\begin{array}{r}\text { [ } 1.3498588075760021 \mathrm{E}+0000 \\
1.3498588075760040 \mathrm{E}+0000]\end{array}$ & $\approx 1.81 \times 10^{-15}$ \\
\hline & 1.0 & $\begin{array}{r}{[1.6487212707001263 \mathrm{E}+0000} \\
1.6487212707001299 \mathrm{E}+0000]\end{array}$ & $\approx 3.54 \times 10^{-15}$ \\
\hline \multirow[t]{3}{*}{$\begin{array}{l}(42) \\
n=4\end{array}$} & 0.2 & $\begin{array}{r}{[1.1051709180756475 \mathrm{E}+0000} \\
1.1051709180756478 \mathrm{E}+0000]\end{array}$ & $\approx 1.60 \times 10^{-16}$ \\
\hline & 0.6 & $\begin{array}{r}\text { [ } 1.3498588075760029 \mathrm{E}+0000 \\
1.3498588075760033 \mathrm{E}+0000]\end{array}$ & $\approx 3.51 \times 10^{-16}$ \\
\hline & 1.0 & $\begin{array}{r}\text { [ } 1.6487212707001277 \mathrm{E}+0000 \\
1.6487212707001285 \mathrm{E}+0000]\end{array}$ & $\approx 7.01 \times 10^{-16}$ \\
\hline
\end{tabular}

decreasing of the number of iterations. These numbers have been equal to 3 for the ABM1 method and to 2 for ABM2, NMS1, and NMS2 methods. For ABM3 and NMS3 methods, only one iteration has been necessary in each step. These results seem to be quite good, taking into account that in conventional predictor-corrector method the state-of-the-art requires a few or several (but not a dozen or so) iterations.

Example 4 In Example 1, we have noticed that the number of steps $n$ and the step size $h$ should be selected suitably for each problem considered. To demonstrate the step size influence on the widths of interval solutions, let us consider once again the problem (60), the NMS3 method and the step sizes $h=0.1,0.01,0.001,0.0001,0.00001,0.000001$. The widths of interval solutions obtained are presented in Fig. 1. From this figure, there is a limit for decreasing the step size, and for too small step sizes, the interval solutions are worse than for larger ones because the larger number of calculations causes a growth of rounding errors. 
Table 6 The solutions of (60) obtained by interval methods of Adams-Moulton type

\begin{tabular}{|c|c|c|c|}
\hline Method & $t=k h \in T k$ & $Y_{k}$ & Width \\
\hline \multirow{4}{*}{$\begin{array}{l}(49) \\
n=1\end{array}$} & 0.2 & [ $1.1051709180755756 \mathrm{E}+0000$ & $\approx 2.26 \times 10^{-13}$ \\
\hline & & $1.1051709180758017 \mathrm{E}+0000]$ & \\
\hline & 0.6 & $\begin{array}{r}\text { [ } 1.3498588075757393 \mathrm{E}+0000 \\
1.3498588075764912 \mathrm{E}+0000]\end{array}$ & $\approx 7.52 \times 10^{-13}$ \\
\hline & 1.0 & $\begin{array}{r}\text { [ } 1.6487212706995912 \mathrm{E}+0000 \\
1.6487212707009854 \mathrm{E}+0000]\end{array}$ & $\approx 1.39 \times 10^{-12}$ \\
\hline \multirow[t]{3}{*}{$\begin{array}{l}(50) \\
n=2\end{array}$} & 0.2 & $\begin{array}{r}\text { [ } 1.1051709180756475 \mathrm{E}+0000 \\
1.1051709180756478 \mathrm{E}+0000]\end{array}$ & $\approx 2.14 \times 10^{-16}$ \\
\hline & 0.6 & $\begin{array}{r}{[1.3498588075760029 \mathrm{E}+0000} \\
1.3498588075760035 \mathrm{E}+0000]\end{array}$ & $\approx 4.90 \times 10^{-16}$ \\
\hline & 1.0 & $\begin{array}{r}1.6487212707001278 \mathrm{E}+0000 \\
1.6487212707001287 \mathrm{E}+0000]\end{array}$ & $\approx 8.37 \times 10^{-16}$ \\
\hline \multirow[t]{3}{*}{$\begin{array}{l}(51) \\
n=3\end{array}$} & 0.2 & $\begin{array}{r}\text { [ } 1.1051709180756475 \mathrm{E}+0000 \\
1.1051709180756478 \mathrm{E}+0000]\end{array}$ & $\approx 1.60 \times 10^{-16}$ \\
\hline & 0.6 & $\begin{array}{r}{[1.3498588075760029 \mathrm{E}+0000} \\
1.3498588075760033 \mathrm{E}+0000]\end{array}$ & $\approx 3.15 \times 10^{-16}$ \\
\hline & 1.0 & 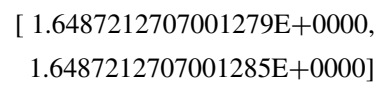 & $\approx 5.20 \times 10^{-16}$ \\
\hline
\end{tabular}

For the same reasons, choosing the optimal step size for a problem, we cannot take too long time integration, i.e., too large value of $a$, where $0 \leq t \leq a$ (the interval $[0, a]$ we denote by $\left.\Delta_{t}\right)$. In [36, Section 3.2], the author considers the initial value problem (60) with $\lambda=-1$ for which the VNODE-LP package with an interval method based on high-order Taylor series [35] yields a very good approximation up to $a=20$ (the width of interval solution obtained is equal to $10^{-6}$ at this point). Such a good approximation for this large value of $a$ cannot be achieved by our methods, which are of rather low orders and use constant step sizes. In Fig. 2, we show how the widths of intervals grow for our NMS3 method with $h=0.0005$ and $t \in[0,20]$. On the other hand, our methods yield as good approximations as other methods of the same order (see the next example).

If for the problem (60) with $\lambda=-1$, we take $a=1$, then the VNODE-LP package produces the output

$$
0.367879441171442[1,6],
$$

which encloses exp(-1). Our ABM3 and NMS3 methods for $h=0.0005$ give the intervals

$$
\text { [ 3.6787944117144198E - 0001, 3.6787944117144263E - 0001] }
$$

and

$$
\text { [ 3.6787944117144211E - 0001, 3.6787944117144248E - 0001] }
$$


Table 7 The solutions of (60) obtained by interval methods of Milne-Simpson type

\begin{tabular}{|c|c|c|c|}
\hline Method & $t=k h \in T_{k}$ & $Y_{k}$ & Width \\
\hline \multirow{4}{*}{$\begin{array}{l}(56) \\
n=1\end{array}$} & 0.2 & [ $1.1051709180753706 \mathrm{E}+0000$ & $\approx 6.78 \times 10^{-13}$ \\
\hline & & $1.1051709180760484 \mathrm{E}+0000]$ & \\
\hline & 0.6 & $\begin{array}{r}1.3498588075751788 \mathrm{E}+0000 \\
1.3498588075774335 \mathrm{E}+0000]\end{array}$ & $\approx 2.25 \times 10^{-12}$ \\
\hline & 1.0 & $\begin{array}{r}1.6487212706987910 \mathrm{E}+0000, \\
1.6487212707029717 \mathrm{E}+0000]\end{array}$ & $\approx 4.18 \times 10^{-12}$ \\
\hline \multirow[t]{3}{*}{$\begin{array}{l}(57) \\
n=2\end{array}$} & 0.2 & $\begin{array}{r}1.1051709180756475 \mathrm{E}+0000 \\
1.1051709180756477 \mathrm{E}+0000]\end{array}$ & $\approx 8.59 \times 10^{-17}$ \\
\hline & 0.6 & $\begin{array}{r}1.3498588075760029 \mathrm{E}+0000 \\
1.3498588075760033 \mathrm{E}+0000]\end{array}$ & $\approx 2.88 \times 10^{-16}$ \\
\hline & 1.0 & $\begin{array}{r}1.6487212707001279 \mathrm{E}+0000 \\
1.6487212707001285 \mathrm{E}+0000]\end{array}$ & $\approx 5.32 \times 10^{-16}$ \\
\hline \multirow[t]{3}{*}{$\begin{array}{l}(58) \\
n=3\end{array}$} & 0.2 & $\begin{array}{r}\text { [ } 1.1051709180756476 \mathrm{E}+0000, \\
1.1051709180756477 \mathrm{E}+0000]\end{array}$ & $\approx 3.06 \times 10^{-17}$ \\
\hline & 0.6 & $\begin{array}{r}1.3498588075760030 \mathrm{E}+0000 \\
1.3498588075760032 \mathrm{E}+0000]\end{array}$ & $\approx 1.00 \times 10^{-16}$ \\
\hline & 1.0 & $\begin{array}{r}{[1.6487212707001280 \mathrm{E}+0000,} \\
1.6487212707001283 \mathrm{E}+0000]\end{array}$ & $\approx 1.84 \times 10^{-16}$ \\
\hline
\end{tabular}

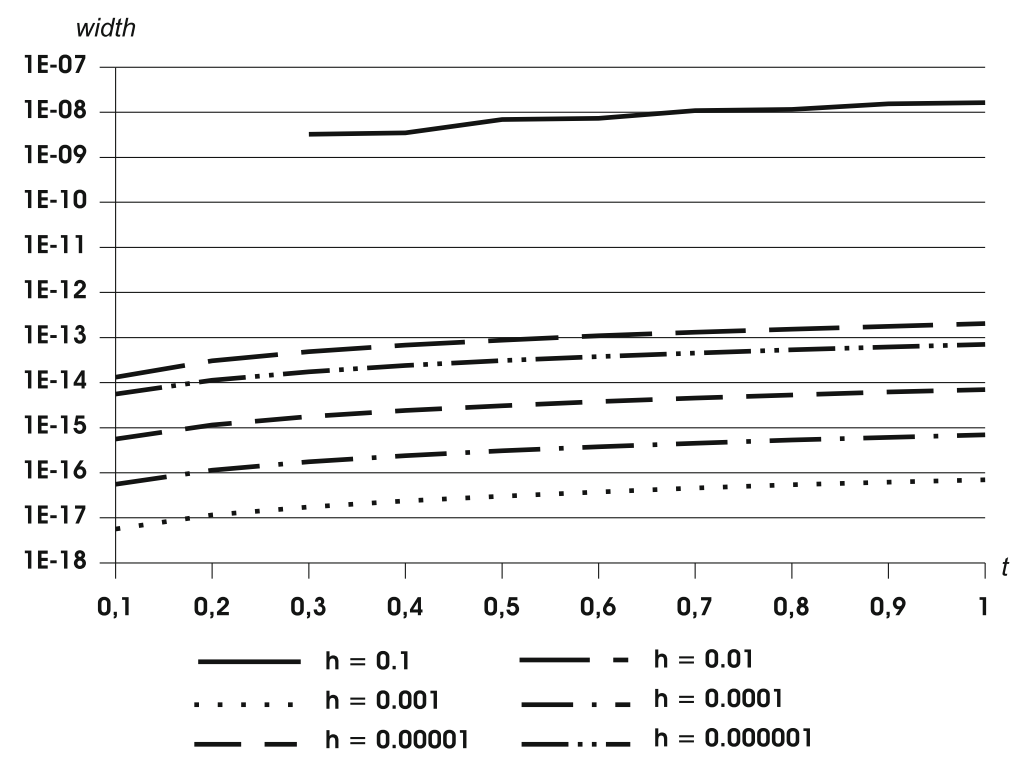

Fig. 1 The widths of interval solutions obtained by the NMS3 method for different step sizes 


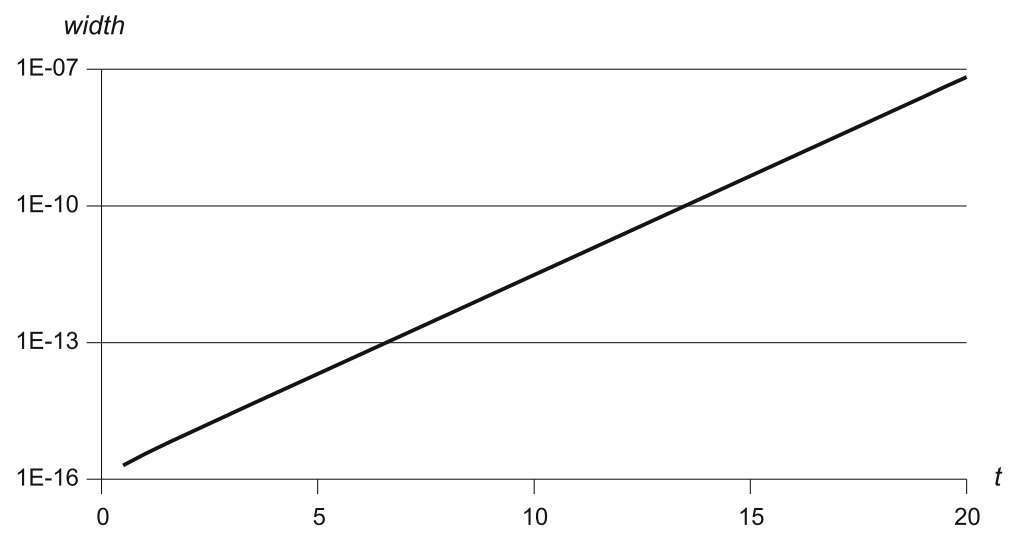

Fig. 2 Growing of interval widths for long time integration

with the widths $6.36 \times 10^{-16}$ and $3.60 \times 10^{-16}$, respectively. In the format used by the VNODE-LP package, these intervals can be written as

$$
0.36787944117144[19,27] \text { and } 0.367879441171442[1,5] \text {, }
$$

and we see that our methods give comparable results (the CPU times are also comparable).

Example 5 Results obtained by our methods may be compared with other interval methods of the same order. Let us take into account the problem A5 from [9, p. 23],

$$
y^{\prime}=\frac{y-t}{y+t}, \quad y(0)=4,
$$

and consider, for instance, the interval explicit Runge-Kutta method of the fourth order (see, e.g., [28, 41]). In interval Runge-Kutta methods, it is necessary to determine a maximum integration interval which guarantees that the interval solutions obtained contains the exact solution. Using the procedure described in [28, 41], and applying this procedure in interval floating-point arithmetic [28] for the initial step size $h_{0}=0.001$, we have found that $t_{\max }$ should be approximately less than 1.46 . Thus, we have taken $h=0.001, \Delta_{t}=[0,1], \Delta_{y}=[4, \overline{4.9}]$, and

$$
\begin{aligned}
& Y_{1}=[\underline{4.0009997500832942}, \overline{4.0009997500832943}], \\
& Y_{2}=[\underline{4.0019990006660423}, \overline{4.0019990006660424}]
\end{aligned}
$$

as additional starting intervals for multistep methods (these intervals have been obtained by the interval explicit Runge-Kutta method of the fourth order with $h=$ 0.0001 ). In Table 8, we present the results obtained at $t=1.0$ by the aforesaid Runge-Kutta method (RK4) and by our ABM3 and NMS3 methods (also of the fourth order). It appears that our NMS3 method gives the intervals with the smallest widths. The programs written by us in Delphi Pascal [29] also show that these methods take significantly less execution time than the considered interval Runge-Kutta method. 
Table 8 Intervals obtained at $t=1$ for the problem (61) by RK4, ABM3, and NM3 methods

\begin{tabular}{lll}
\hline Method & $Y$ & Width \\
\hline RK4 & {$[4.8075923778847051,4.8075923778847063]$} & $\approx 1.07 \times 10^{-15}$ \\
ABM3 & {$[4.8075923778847056,4.8075923778847069]$} & $\approx 1.18 \times 10^{-15}$ \\
NMS3 & {$[4.8075923778847059,4.8075923778847067]$} & $\approx 7.23 \times 10^{-16}$ \\
\hline
\end{tabular}

Multistep interval predictor-corrector methods can be also used for solving more than one-dimensional problems.

Example 6 Let us consider the motion of a simple pendulum described by

$$
\varphi^{\prime \prime}+u^{2} \sin \varphi=0
$$

where $\varphi=\varphi(t), u=\sqrt{g / L}, g$ is the gravitational acceleration at the Earth's surface, and $L$ denotes the pendulum length. If we assume that the angle $\varphi$ is small, i.e., $\sin \varphi \approx \varphi,{ }^{3}$ then the (62) can be reduced to the equation of simple harmonic motion

$$
\varphi^{\prime \prime}+u^{2} \varphi=0,
$$

with the solution $\varphi(t)=\varphi_{0} \cos (u t)$, where $\varphi_{0}$ is an initial angle. Denoting $y_{1}=\varphi^{\prime}$, $y_{2}=\varphi$, and assuming that $\varphi^{\prime}(0)=0, \varphi(0)=0$, we can transform (63) into the following systems of differential equations of the first order:

$$
y_{1}^{\prime}=-u^{2} y_{2}, \quad y_{2}^{\prime}=y_{1}
$$

with the initial conditions

$$
y_{1}(0)=0, \quad y_{2}(0)=\varphi_{0} .
$$

For $g=9.80665, L=1$, and $\varphi_{0}=\pi / 6$, the exact solution at selected points $t$ is presented in Table 9.

Assuming additional starting intervals as follows:

$$
\begin{aligned}
& Y_{1}=[\underline{-0.00513474154027124}, \overline{-0.00513474154027123}], \\
& Y_{2}=\left[\begin{array}{ll}
\underline{0.52359620822533077}, & \overline{0.52359620822533078}
\end{array}\right]
\end{aligned}
$$

and applying interval predictor-corrector methods ABM2 and NMS2 we have obtained (after about 5 corrector iterations in each step) interval solutions presented in Tables 10 and 11. In both of these interval methods, we assumed $g=[\underline{9.80665}, \overline{9.80665}]$, the accuracy $10^{-18}$ in the iterations, and took $h=0.001$,

$$
\Delta_{t}=[0,2], \quad \Delta_{y_{1}}=[\underline{-1.8}, \overline{1.8}], \quad \Delta_{y_{2}}=[\underline{-0.6}, \overline{0.6}] .
$$

We can observe that for each $t$ the exact solution belongs to the interval solutions obtained and that the NMS2 method gives a somewhat better interval solution than the ABM2 method.

\footnotetext{
${ }^{3}$ For $|\varphi|<5^{\circ}$ we have $|\sin \varphi-\varphi|<1.1 \cdot 10^{-4}$.
} 
Table 9 The approximate (with 15 digits after decimal point) exact solution of (64)-(65)

\begin{tabular}{lrr}
\hline$t$ & \multicolumn{1}{l}{$y_{1}(t)$} & \multicolumn{1}{l}{$y_{2}(t)$} \\
\hline 0.5 & -1.639658832231953 & 0.002627285350445 \\
1.0 & -0.016454781143167 & -0.523572409500308 \\
1.5 & 1.639493700417002 & -0.007881591454600 \\
2.0 & 0.032907905107624 & 0.523493313861694
\end{tabular}

Solving any problem by any interval method (not only by those considered in this paper), we should keep the wrapping effect in mind (see, e.g., [13]). This effect sometimes leads to a sudden expansion of interval widths.

Example 7 As it is well-known, the motion of the Moon in a rotating coordinate system on a plane is described by the Hill equations of the form (a nonstiff problem):

$$
\begin{aligned}
\frac{d y_{l}}{d \tau} & =y_{l+2}, \quad l=1,2, \\
\frac{d y_{3}}{d \tau} & =2 M y_{4}-\left(\frac{\kappa}{r^{3}}-3 M^{2}\right) y_{1}, \\
\frac{d y_{4}}{d \tau} & =-2 M y_{3}-\frac{\kappa}{r^{3}} y_{2}, \\
y_{l}\left(\tau_{0}\right) & =y_{l}^{0}, \quad l=1,2,3,4,
\end{aligned}
$$

\begin{tabular}{|c|c|c|}
\hline$t_{k} \in T_{k}$ & $Y_{s}=Y_{s k}$ & Width of $Y_{s}$ \\
\hline \multirow[t]{2}{*}{0.5} & $\begin{aligned} Y_{1}= & {[-1.6396588322973040 \mathrm{E}+0000} \\
& -1.6396588321658868 \mathrm{E}+0000]\end{aligned}$ & $\approx 1.31 \times 10^{-10}$ \\
\hline & $Y_{2}=\left[\begin{array}{l}2.6272853324493007 \mathrm{E}-0003 \\
2.6272853741242819 \mathrm{E}-0003]\end{array}\right.$ & $\approx 4.17 \times 10^{-11}$ \\
\hline \multirow[t]{2}{*}{1.0} & $\begin{aligned} Y_{1}= & {[-1.6454781633306984 \mathrm{E}-0002} \\
& -1.6454780688655671 \mathrm{E}-0002]\end{aligned}$ & $\approx 9.45 \times 10^{-10}$ \\
\hline & $\begin{aligned} Y_{2}=[ & -5.2357240965081014 \mathrm{E}-0001 \\
& -5.2357240934949198 \mathrm{E}-0001]\end{aligned}$ & $\approx 3.01 \times 10^{-10}$ \\
\hline \multirow[t]{2}{*}{1.5} & $\begin{aligned} Y_{1}=\left[\begin{array}{l}1.6394936974312226 \mathrm{E}+0000 \\
1.6394937034213517 \mathrm{E}+0000\end{array}\right]\end{aligned}$ & $\approx 5.99 \times 10^{-9}$ \\
\hline & $\begin{aligned} Y_{2}= & {[-7.8815924193757663 \mathrm{E}-0003} \\
& -7.8815905068929912 \mathrm{E}-0003]\end{aligned}$ & $\approx 1.91 \times 10^{-9}$ \\
\hline \multirow[t]{2}{*}{2.0} & $Y_{1}=\left[\begin{array}{l}3.2907886495247670 \mathrm{E}-0002 \\
3.2907923791272733 \mathrm{E}-0002]\end{array}\right.$ & $\approx 3.73 \times 10^{-8}$ \\
\hline & $Y_{2}=\left[\begin{array}{l}5.2349330790666918 \mathrm{E}-0001 \\
5.2349331981606120 \mathrm{E}-0001]\end{array}\right.$ & $\approx 1.19 \times 10^{-8}$ \\
\hline
\end{tabular}

Table 10 The interval solution of (64)-(65) obtained by the ABM2 method 
Table 11 The interval solution of (64)-(65) obtained by the NMS2 method

\begin{tabular}{|c|c|c|}
\hline$t_{k} \in T_{k}$ & $Y_{s}=Y_{s k}$ & Width of $Y_{s}$ \\
\hline \multirow[t]{2}{*}{0.5} & $\begin{aligned} Y_{1}=[ & -1.6396588322877012 \mathrm{E}+0000 \\
& -1.6396588321757556 \mathrm{E}+0000]\end{aligned}$ & $\approx 1.12 \times 10^{-10}$ \\
\hline & $Y_{2}=\left[\begin{array}{l}2.6272853323378610 \mathrm{E}-0003 \\
2.6272853677648693 \mathrm{E}-0003]\end{array}\right.$ & $\approx 3.54 \times 10^{-11}$ \\
\hline \multirow[t]{2}{*}{1.0} & $\begin{aligned} Y_{1}=[ & -1.6454781463064509 \mathrm{E}-0002 \\
& -1.6454780817520932 \mathrm{E}-0002]\end{aligned}$ & $\approx 6.46 \times 10^{-10}$ \\
\hline & $\begin{aligned} Y_{2}=[ & -5.2357240960311741 \mathrm{E}-0001 \\
& -5.2357240939736414 \mathrm{E}-0001]\end{aligned}$ & $\approx 2.06 \times 10^{-10}$ \\
\hline \multirow[t]{2}{*}{1.5} & $Y_{1}=\left[\begin{array}{l}1.6394936988275034 \mathrm{E}+0000 \\
1.6394937020266249 \mathrm{E}+0000]\end{array}\right.$ & $\approx 3.20 \times 10^{-9}$ \\
\hline & $\begin{aligned} Y_{2}=[ & -7.8815919638709175 \mathrm{E}-0003 \\
& -7.8815909426989587 \mathrm{E}-0003]\end{aligned}$ & $\approx 1.02 \times 10^{-9}$ \\
\hline \multirow[t]{2}{*}{2.0} & $Y_{1}=\left[\begin{array}{l}3.2907897391532022 \mathrm{E}-0002 \\
3.2907912813019946 \mathrm{E}-0002]\end{array}\right.$ & $\approx 1.54 \times 10^{-8}$ \\
\hline & $Y_{2}=\left[\begin{array}{l}5.2349331139958767 \mathrm{E}-0001 \\
5.2349331632372242 \mathrm{E}-0001]\end{array}\right.$ & $\approx 4.92 \times 10^{-9}$ \\
\hline
\end{tabular}

where

$$
r=\sqrt{y_{1}^{2}+y_{2}^{2}}, \quad \tau=\left(v-v^{\prime}\right)\left(t-t_{0}\right), \quad M=\frac{v^{\prime}}{v-v^{\prime}}, \quad \kappa=G \frac{m_{0}+m_{1}}{\left(v-v^{\prime}\right)^{2}},
$$

and where $v$ is the mean motion of the Moon, $v^{\prime}$ is the mean motion of the $\operatorname{Sun}, G$ is the gravitational constant, $m_{0}$ is the mass of the Earth, $m_{1}$ is the mass of the Moon, and $t_{0}$ is an initial moment. If we take $M=0, \kappa=1$, and assume that at $\tau_{0}=0$, we have $y_{1}(0)=y_{4}(0)=1, y_{2}(0)=y_{3}(0)=0$. Then the initial value problem (66) has the solution

$$
y_{1}(\tau)=y_{4}(\tau)=\cos \tau, \quad y_{2}(\tau)=\sin \tau, \quad y_{3}(\tau)=-\sin \tau
$$

The numerical values of the above solution at some moments are given in Table 12 .

To solve problem (66) by an interval predictor-corrector method, we have used the NMS3 method with $h=0.0005$. Additional staring intervals are presented in Table 13.

Table 12 The approximate (with 15 digits after decimal point) exact solution of (66) with $M=0$ and $\kappa=1$

\begin{tabular}{lrr}
\hline$\tau$ & $y_{1}(\tau)=y_{4}(\tau)$ & \multicolumn{1}{l}{$y_{2}(\tau)=-y_{3}(\tau)$} \\
\hline 1.0 & 0.540302305868140 & 0.841470984807897 \\
3.0 & -0.989992496600446 & 0.141120008059867 \\
5.0 & 0.283662185463226 & -0.958924274663139 \\
\hline
\end{tabular}


Table 13 Starting intervals for NMS3 method and the problem (66) with $M=0$ and $\kappa=1$

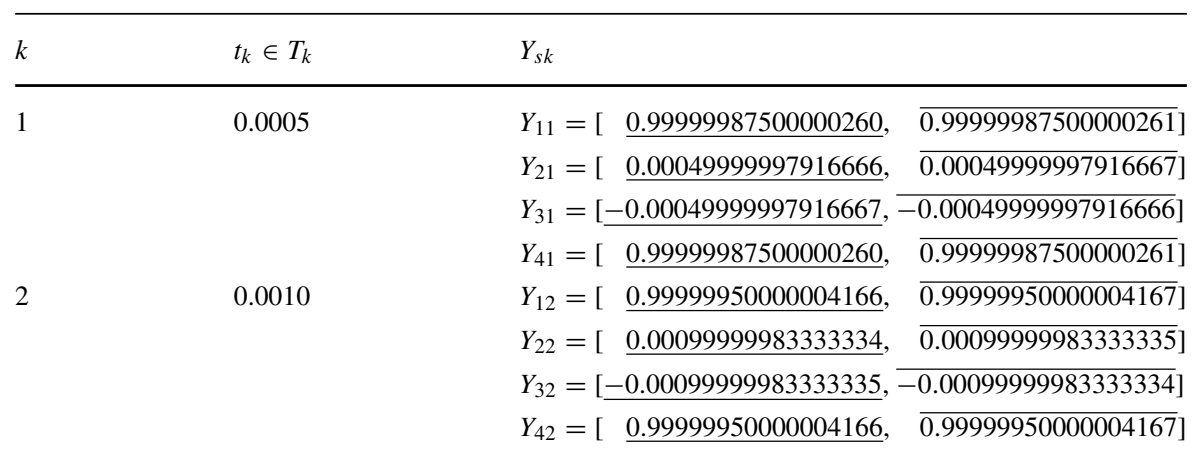

Let us note that we cannot take the whole domains of definitions of $y_{s}$, where $s=1,2$, to determine $\triangle_{y_{s}}$, because we would get divisions by intervals containing zero. For

$\Delta_{t}=[0, \overline{1.001}], \quad \Delta_{y_{1}}=\Delta_{y_{4}}=[\underline{0.4}, \overline{1.1}], \quad \Delta_{y_{2}}=[\underline{-0.1}, 1], \quad \Delta_{y_{3}}=[-1, \overline{0.1}]$

we can find the interval solutions at $t=1$, and additionally at $t=1.0005$ and $t=1.0010$. The intervals obtained for these values of $t$ can be used as new starting points. Taking

$\Delta_{t}=[1, \overline{2.001}], \quad \Delta_{y_{1}}=\Delta_{y_{4}}=[\underline{-0.5}, \overline{0.6}], \quad \Delta_{y_{2}}=[\underline{0.8}, \overline{1.1}], \quad \Delta_{y_{3}}=[-1, \overline{-0.7}]$

we obtain the interval solution at $t=2$, and additionally, at $t=2.0005$ and $t=2.0010$. Proceeding further in the same way and assuming that

$$
\Delta_{y_{1}}=\Delta_{y_{4}}=[\underline{-1.1}, \overline{-0.3}], \quad \Delta_{y_{2}}=[0,1], \quad \Delta_{y_{3}}=[-1,0]
$$

for $t \in[2,3.001]$,

$$
\Delta_{y_{1}}=\Delta_{y_{4}}=[-1, \overline{0.6}], \quad \Delta_{y_{2}}=[\underline{-0.9}, \overline{0.2}], \quad \Delta_{y_{3}}=[\underline{-0.2}, \overline{0.9}]
$$

for $t \in[3,4.001]$ and

$$
\Delta_{y_{1}}=\Delta_{y_{4}}=[\underline{-0.6}, \quad \overline{0.4}], \quad \Delta_{y_{2}}=[-1, \overline{-0.7}], \quad \Delta_{y_{3}}=[\underline{0.7}, 1]
$$

for $t \in[4,5.001]$, it is possible to get the interval solutions up to $t=5.001$. In Table 14, we present the interval solutions obtained at $t=1.0,3.0,5.0$. The number of corrector iterations does not exceed 4 in each step. Unfortunately, for $t$ approximately greater than 16 the widths of intervals have been greater than $10^{-2}$, and the interval solution is worthless, but we do not observe the wrapping effect. It should be noted that using the explicit method of Adams-Bashforth type with $n=4$, i.e., using the method (36), we have the wrapping effect for our problem already for $t$ approximately greater than 5 (see Fig. 3). Of course, in each case, the exact solution belongs to the interval solution obtained (compare Table 12). 
Table 14 The interval solution of (66) obtained by the NMS3 method

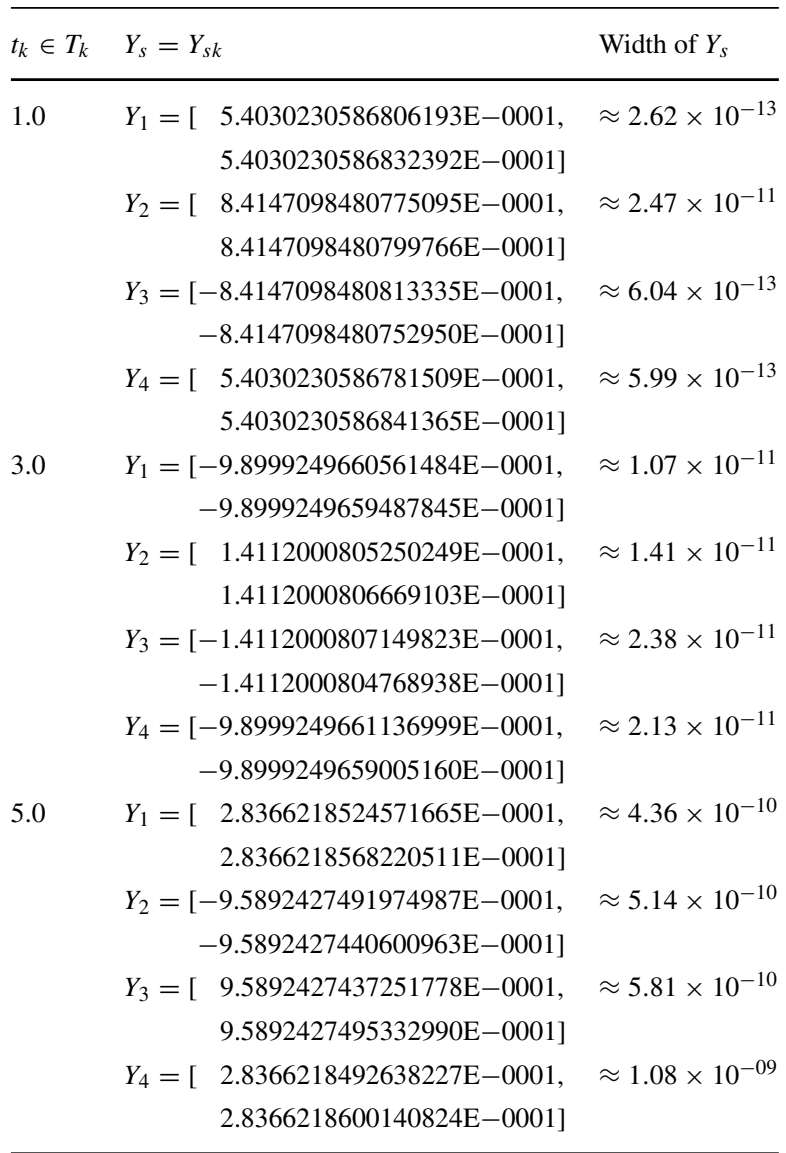

Example 8 Let us consider a stiff problem. Let us take the initial value problem E2 given in [8, p. 32] and [9, p. 21], i.e.,

$$
\begin{gathered}
y_{1}^{\prime}=y_{2}, \quad y_{2}^{\prime}=5\left(1-y_{1}^{2}\right) y_{2}-y_{1}, \\
y_{1}(0)=2, \quad y_{2}(0)=0 .
\end{gathered}
$$

Assuming

$$
\Delta_{t}=\{t \in \mathbb{R}: 0 \leq t \leq 1\},
$$

$$
\Delta_{y_{1}}=\left\{y_{1} \in \mathbb{R}: 1.8 \leq y_{1} \leq 2.1\right\}, \quad \Delta_{y_{2}}=\left\{y_{2} \in \mathbb{R}:-0.2 \leq y_{2} \leq 0.1\right\},
$$

$h=0.0001$, and taking additional starting intervals given in Table 15, we have obtained by our NMS3 method the results presented in Table 16 (with the number of iterations not greater then 3 ).

Since implicit methods are recognized as appropriate for stiff differential equations (see, e.g., [5] and [16]), to compare our method to another one of the same order, let us consider an implicit interval Runge-Kutta method. From a variety of such methods of fourth order, let us choice the interval version of Butcher's threestage semi-implicit method [6, p. 51] and [15, p. 205] presented (among others) in 


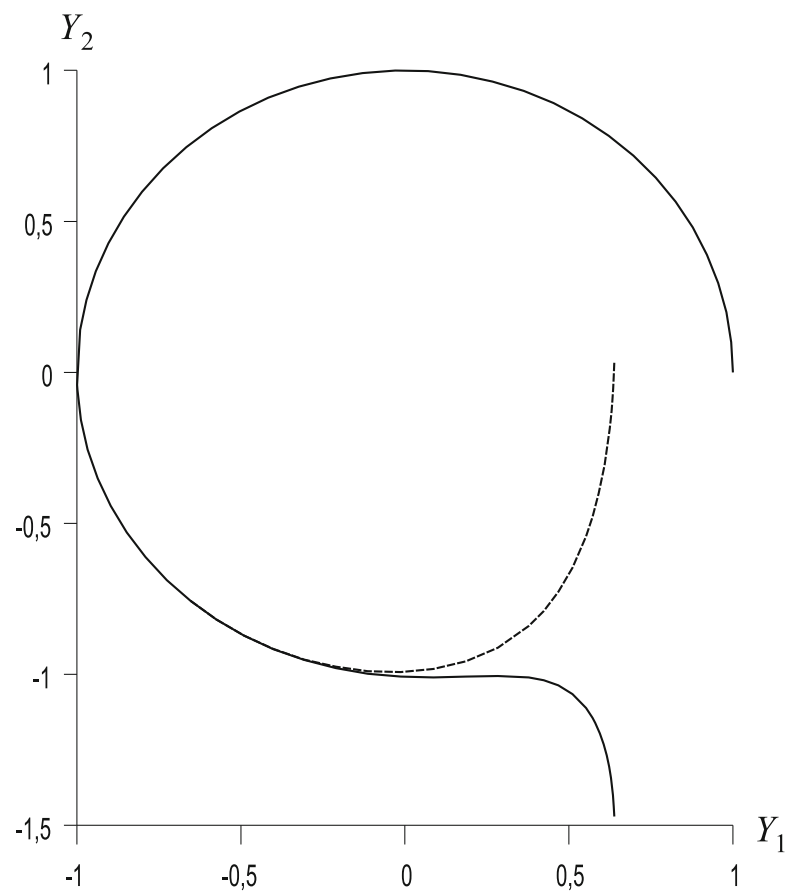

Fig. 3 The wrapping effect caused by the method (36) for the problem (66) with $M=0$ and $\kappa=1$

[28, p. 79]. In this method, a few of coefficients are equal to zero, significantly reducing the number of calculations. As we mentioned in Example 5, from the theory of interval Runge-Kutta method, it appears that it is necessary to determine maximum integration interval. For the problem (67), $h=0.0001$ and regions (68), we have obtained $t_{\max } \leq 0.053$ approximately. Using the same data as previously, at $t=0.05$ by the interval version of Butcher's method, we get

$$
\begin{aligned}
& Y_{1}=[1.9980234267738453 \mathrm{E}+0000, \quad 1.9980234267738455 \mathrm{E}+0000], \\
& Y_{2}=[-7.0355564016027554 \mathrm{E}-0002,-7.0355564016027261 \mathrm{E}-0002] \text {, }
\end{aligned}
$$

\begin{tabular}{|c|c|c|}
\hline$k$ & $t=t h \in T_{k}$ & $Y_{s}=Y_{s k}$ \\
\hline 1 & 0.0001 & 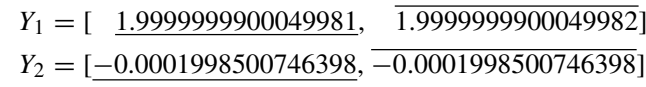 \\
\hline 2 & 0.0002 & 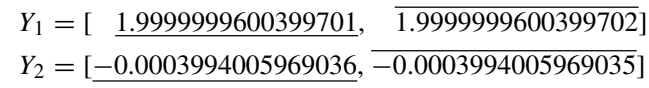 \\
\hline
\end{tabular}

Table 15 Starting intervals for the NMS3 method and the problem (67) 
Table 16 The interval solution of (67) obtained by the NMS3 method

\begin{tabular}{|c|c|c|}
\hline$t_{k} \in T_{k}$ & $Y_{s}=Y_{s k}$ & Width of $Y_{s}$ \\
\hline \multirow[t]{2}{*}{0.2} & $Y_{1}=\left[\begin{array}{l}1.9817243912807375 \mathrm{E}+0000 \\
1.9817243912807382 \mathrm{E}+0000]\end{array}\right.$ & $\approx 6.40 \times 10^{-16}$ \\
\hline & $\begin{aligned} Y_{2}= & {[-1.2774153125696619 \mathrm{E}-0001,} \\
& -1.2774153125695947 \mathrm{E}-0001]\end{aligned}$ & $\approx 6.71 \times 10^{-15}$ \\
\hline \multirow[t]{2}{*}{0.6} & $Y_{1}=\left[\begin{array}{l}1.9271919568429724 \mathrm{E}+0000 \\
1.9271919568431276 \mathrm{E}+0000]\end{array}\right.$ & $\approx 1.55 \times 10^{-13}$ \\
\hline & $\begin{aligned} Y_{2}= & {[-1.4072022721993012 \mathrm{E}-0001,} \\
& -1.4072022721776137 \mathrm{E}-0001]\end{aligned}$ & $\approx 2.17 \times 10^{-12}$ \\
\hline \multirow[t]{2}{*}{1.0} & $Y_{1}=\left[\begin{array}{l}1.8694388533760272 \mathrm{E}+0000 \\
1.8694388534102298 \mathrm{E}+0000]\end{array}\right.$ & $\approx 4.42 \times 10^{-11}$ \\
\hline & $\begin{aligned} Y_{2}= & {[-1.4823587559892300 \mathrm{E}-0001} \\
& -1.4823587515535485 \mathrm{E}-0001]\end{aligned}$ & $\approx 4.44 \times 10^{-10}$ \\
\hline
\end{tabular}

with widths $1.23 \times 10^{-16}$ and $2.93 \times 10^{-16}$, respectively, while our NMS3 method yields

$$
\begin{aligned}
& Y_{1}=\left[\begin{array}{rr}
1.9980234267738453 \mathrm{E}+0000, & 1.9980234267738455 \mathrm{E}+0000
\end{array}\right], \\
& Y_{2}=[-7.0355564016027461 \mathrm{E}-0002,-7.0355564016026966 \mathrm{E}-0002],
\end{aligned}
$$

with similar widths, namely $1.50 \times 10^{-16}$ and $4.94 \times 10^{-16}$, respectively. On the other hand, the VNODE-LP package (based on high-order Taylor series) gives

$$
Y_{1}=1.99802342677384[48,55], \quad Y_{2}=-0.070355564016027[1,3] .
$$

Comparing all these results, we see that the NMS3 method yields comparable approximations to the solution.

In all previous examples, the initial values have been point intervals, and additional starting intervals have been very tiny. But our methods can be also applied in the case of data uncertainties.

Example 9 Let us consider the same differential equations as in the previous example, the same regions $\Delta_{t}, \Delta_{y_{1}}, \Delta_{y_{2}}$, the same step size $h=0.0001$, but now let us assume that

$$
\begin{gathered}
Y_{1}(0)=Y_{1}(0.0001)=Y_{1}(0.0002)=[\underline{1.9999}, \overline{2.0001}] \\
Y_{2}(0)=[\overline{-0.0001}, \overline{0.0001}], \\
Y_{2}(0.0001)=\left[\underline{-0.0003}, \overline{-0.0001], \quad Y_{2}}(0.0002)=[\underline{-0.0005}, \overline{-0.0003}] .\right.
\end{gathered}
$$

(In program presented in [30] for this example, one can take other starting intervals.) The results obtained for these data by the NMS3 method are presented in Table 17. We see that after 3000 steps, i.e., for $t=0.3$, the results are quite good. For greater $t$, the results are rather less valuable (for instance, for $t=0.7$ the widths of intervals are equal to 1 , approximately). 
Table 17 The interval solution of (67) with starting intervals (69) obtained by the NMS3 method

\begin{tabular}{|c|c|c|}
\hline$t_{k} \in T_{k}$ & $Y_{s}=Y_{s k}$ & Width of $Y_{s}$ \\
\hline \multirow[t]{2}{*}{0.1} & $Y_{1}=\left[\begin{array}{l}1.9934447874234045 \mathrm{E}+0000 \\
1.9936944029757450 \mathrm{E}+0000\end{array}\right]$ & $\approx 2.50 \times 10^{-4}$ \\
\hline & $\begin{aligned} Y_{2}= & {[-1.0421455291082735 \mathrm{E}-0001,} \\
& -1.0322295075061154 \mathrm{E}-0001]\end{aligned}$ & $\approx 9.92 \times 10^{-4}$ \\
\hline \multirow[t]{2}{*}{0.2} & $Y_{1}=\left[\begin{array}{l}1.9814818734815870 \mathrm{E}+0000 \\
1.9819670193640089 \mathrm{E}+0000\end{array}\right]$ & $\approx 4.85 \times 10^{-4}$ \\
\hline & $\begin{aligned} Y_{2}= & {[-1.3002936343647112 \mathrm{E}-0001} \\
& -1.2545436653063669 \mathrm{E}-0001]\end{aligned}$ & $\approx 4.57 \times 10^{-3}$ \\
\hline \multirow[t]{2}{*}{0.3} & $Y_{1}=\left[\begin{array}{l}1.9677925092657343 \mathrm{E}+0000 \\
1.9693308620296675 \mathrm{E}+0000]\end{array}\right.$ & $\approx 1.54 \times 10^{-3}$ \\
\hline & $\begin{aligned} Y_{2}= & {[-1.4438756137265273 \mathrm{E}-0001} \\
& -1.2424631038446131 \mathrm{E}-0001]\end{aligned}$ & $\approx 2.01 \times 10^{-2}$ \\
\hline
\end{tabular}

\section{Conclusions}

The main conclusion from the examples presented and many others carried out by the authors is that the interval methods executed in floating-point interval arithmetic yield approximations of solutions in the form of intervals which contain all possible numerical errors, i.e., representation errors, rounding errors, truncation errors, and also data uncertainties. Other conclusions concerning the multistep interval methods are as follows:

- for the same number of steps explicit interval methods of Nyström type are somewhat better (give intervals with smaller widths) than the methods of Adams-Bashforth type,

- for the same number of steps implicit interval methods of Milne-Simpson type give somewhat better results (with smaller widths) than the methods of AdamsMoulton type,

- the application of an explicit interval multistep method as the predictor for an implicit one significantly reduces the number of iterations involved (in comparison with the case when in an implicit interval method the solution for the previous step is taken as the initial approximation),

- for each problem one should choose the appropriate step size or the number of method steps to obtain the interval solution with the smallest width (for a given step size there exists the optimal number of method steps, and for a given number of method steps there exists the best step size).

The examples presented in this paper clearly show that the interval predictorcorrector methods can be applied successfully to both nonstiff and stiff problems, and to both one- and more than one-dimensional problems (see Examples 6-8). Comparing the results with other interval methods of the same or similar order, we see the high compatibility of the interval solutions obtained. Although our methods are of low order, in the case when the integration intervals are not too big they give as good 
results as the methods based on high-order Taylor series. Undoubtedly, an advantage of our methods consists in their simplicities - they can be easy programmed in any computer language. We hope that an implementation of variable step size controls would benefit in speed for these methods. We plan to study this problem in our next works.

Acknowledgments The paper was supported by the Poznan University of Technology (Poland) through Grants No. 09/91/DSPB/0600 and 02/21/DSPB/3477.

Open Access This article is distributed under the terms of the Creative Commons Attribution 4.0 International License (http://creativecommons.org/licenses/by/4.0/), which permits unrestricted use, distribution, and reproduction in any medium, provided you give appropriate credit to the original author(s) and the source, provide a link to the Creative Commons license, and indicate if changes were made.

\section{References}

1. Alefeld, G., Herzberger, J.: Introduction to Interval Computations. Academic Press, New York (1983)

2. Bauch, H.: On the iterative inclusion of solutions in initial-value problems for ordinary differential equations. Computing 22, 339-354 (1979)

3. Berz, M., Hoffstätter, G.: Computation and application of taylor polynomials with interval remainder bounds. Reliab. Comput. 4(1), 83-97 (1998)

4. Berz, M., Makino, K.: Performance of Taylor model methods for validated integration of ODEs. In: Dongarra, J., Madsen, K., Wasniewski, J. (eds.) Applied Parallel Computing. State of the Art in Scientific Computing, Lecture Notes in Computer Science, vol. 3732, pp. 65-73 (2005)

5. Butcher, J.C.: Implicit Runge-Kutta processes. Math. Comput. 18, 50-64 (1964)

6. Butcher, J.C.: The Numerical Analysis of Ordinary Differential Equations: Runge-Kutta and General Linear Methods. Wiley, Chichester (1987)

7. Corliss, G.F., Rihm, R.: Validating an a priori enclosure using high-order Taylor series. In: In Scientific Computing, Computer Arithmetic, and Validated Numerics, pp. 228-238. Akademie Verlag (1996)

8. Enright, W.H., Hull, T.R., Lindberg, B.: Comparing numerical methods for stiff systems of O.D.E:s. BIT Numer. Math. 15(1), 10-48 (1975)

9. Enright, W.H., Pryce, J.D.: Two fortran packages for assessing initial value methods. ACM Trans. Math. Softw. 13(1), 1-27 (1987)

10. Gajda, K., Jankowska, M., Marciniak, A., Szyszka, B.: A survey of interval Runge-Kutta and multistep methods for solving the initial value problem. In: Wyrzykowski, R., Dongarra, J., Karczewski, K., Wasniewski, J. (eds.) Parallel Processing and Applied Mathematics, Lecture Notes in Computer Science, vol. 4967, pp. 1361-1371. Springer, Berlin (2008)

11. Gajda, K., Marciniak, A., Szyszka, B.: Three-and four-stage implicit interval methods of Runge-Kutta type. Computational Methods in Science and Technology 6(1), 41-59 (2000)

12. Gambill, T.N., Skeel, R.D.: Logarithmic reduction of the wrapping effect with application to ordinary differential equations. SIAM J. Numer. Anal. 25(1), 153-162 (1988)

13. Hammer, R., Hocks, M., Kulisch, U., Ratz, D.: Numerical Toolbox for Verified Computing I. Basic Numerical Problems, Theory, Algorithms, and Pascal-XSC Programs. Springer, Berlin (1993)

14. Hansen, E.R.: Topics in Interval Analysis. Oxford University Press, London (1969)

15. Heirer, E., Nrsett, S.P., Wanner, G.: Solving Ordinary Differential Equations I-Nonstiff Problems. Springer, Berlin (1987)

16. Heirer, E., Wanner, G.: Solving Ordinary Differential Equations II-Stiff and Differential-Algebraic Problems. Springer, Berlin (1991)

17. Jackson, K.R., Nedialkov, N.S.: Some recent advances in validated methods for IVPs for ODEs. Appl. Numer. Math. 42(1-3), 269-284 (2002)

18. Jankowska, M., Marciniak, A.: Implicit interval methods for solving the initial value problem. Computational Methods in Science and Technology 8(1), 17-30 (2002) 
19. Jankowska, M., Marciniak, A.: On explicit interval methods of Adams-Bashforth type. Computational Methods in Science and Technology 8(2), 46-57 (2002)

20. Jankowska, M., Marciniak, A.: On two families of implicit interval methods of Adams-Moulton type. Computational Methods in Science and Technology 12(2), 109-113 (2006)

21. Kalmykov, S.A., Shokin, J.I., Juldashev, E.C.: Solving Ordinary Differential Equations by Interval Methods. In: Doklady AN SSSR, vol. 230 (1976)

22. Lohner, R.J.: Computation of guaranteed enclosures for the solutions of ordinary initial and boundary value problems. In: Cash, J.R., Gladwell, I. (eds.) Computational Ordinary Differential Equations, pp. 425-435. Clarendon Press, Oxford (1992)

23. Makino, K., Berz, M.: Suppression of the wrapping effect by taylor Model-Based verified integrators: long-term stabilization by preconditioning. International Journal of Differential Equations and Applications 10(4), 353-384 (2005)

24. Makino, K., Berz, M.: Suppression of the wrapping effect by taylor Model-Based verified integrators: the single step. International Journal of Pure and Applied Mathematics 36(2), 175-197 (2006)

25. Marciniak, A.: Implicit interval methods for solving the initial value problem. Numerical Algorithms 37(1-4), 241-251 (2004)

26. Marciniak, A.: Multistep interval methods of nyström and Milne-Simpson types. Computational Methods in Science and Technology 13(1), 23-40 (2007)

27. Marciniak, A.: On multistep interval methods for solving the initial value problem. J. Comput. Appl. Math. 199(2), 229-237 (2007)

28. Marciniak, A.: Selected Interval Methods for Solving the Initial Value Problem. Publishing House of Poznan University of Technology, Poznan (2009)

29. Marciniak, A.: Delphi Pascal Programs for Interval Multistep Methods (2016). http://www.cs.put. poznan.pl/amarciniak/IPCM-Examples

30. Marciniak, A.: Interval Arithmetic Unit (2016). http://www.cs.put.poznan.pl/amarciniak/IAUnits/ IntervalArithmetic32and64.pas

31. Marciniak, A., Szyszka, B.: One-and two-stage implicit interval methods of Runge-Kutta type. Computational Methods in Science and Technology 5(1), 53-65 (1999)

32. Moore, R.E.: The Automatic Analysis and Control of Error in Digital Computation Based on the Use of Interval Numbers. In: Rall, L.B. (ed.) Error in Digital Computation, vol. 1, pp. 61-130. Wiley, New York (1965)

33. Moore, R.E.: Interval Analysis. Prentice-Hall, Englewood Cliffs (1966)

34. Moore, R.E.: Methods and Applications of Interval Analysis. SIAM Society for Industrial \& Applied Mathematics, Philadelphia (1979)

35. Nedialkov, N.S.: Interval Tools for ODEs and DAEs. Tech. Rep. CAS 06-09-NN, Department of Computing and Software, McMaster University, Hamilton (2006)

36. Nedialkov, N.S.: VNODE-LP — a Validated Solver for Initial Value Problems in Ordinary Differential Equations. Tech. Rep. CAS 06-06-NN, Department of Computing and Software, McMaster University, Hamilton (2006)

37. Nedialkov, N.S., Jackson, K.R.: A New Perspective on the Wrapping Effect in Interval Methods for Initial Value Problems for Ordinary Differential Equations. In: Facius, A., Kulisch, U., Lohner, R. (eds.) Perspectives on Enclosure Methods, pp. 219-264. Springer, Vienna (2001)

38. Nedialkov, N.S., Jackson, K.R., Corliss, G.F.: Validated solutions of initial value problems for ordinary differential equations. Appl. Math. Comput. 105(1), 21-68 (1999)

39. Nickel, K.: How to Fight the Wrapping Effect. In: Goos, G., Hartmanis, J. (eds.) Lecture Notes in Computer Science, pp. 121-132. Springer, Berlin (1986)

40. Nickel, K.: Using interval methods for the numerical solution of ODE's. ZAMM-Journal of Applied Mathematics and Mechanics/Zeitschrift fü,r Angewandte Mathematik und Mechanik 66(11), 513-523 (1986)

41. Shokin, Y.I.: Interval Analysis. Novosibirsk, Nauka (1981)

42. Stewart, N.F.: A heuristic to reduce the wrapping effect in the numerical solution of $x=f(t, x)$. BIT Numer. Math. 11(3), 328-337 (1971) 